\title{
Vertical Syndication-Proof Competitive Prices in Multilateral Assignment Markets
}

\author{
O. Tejada*1 and M. Álvarez-Mozos ${ }^{2}$ \\ ${ }^{1}$ CER-ETH Center of Economic Research, ETH Zurich, Switzerland \\ ${ }^{2}$ Departament de Matemàtica Econòmica, Financera $i$ Actuarial \\ Universitat de Barcelona, Spain. \\ Published in Review of Economic Design (2016) \\ Published version available at http://link.springer.com \\ DOI: $10.1007 / \mathrm{s} 10058-016-0193-1$
}

\begin{abstract}
We consider a market comprising a number of perfectly complementary and homogeneous commodities. We concentrate on the incentives for firms producing these commodities to merge and form a vertical syndicate. The main result establishes that the nucleolus of the associated market game corresponds to the unique vector of prices with the following properties: (i) they are vertical syndication-proof, (ii) they are competitive, (iii) they yield the average of the buyersand the sellers-optimal allocations in bilateral markets, and (iv) they depend on the traders' bargaining power but not on their identity. The proof uses an isomorphism between our class of market games and the entire class of bankruptcy games.

Keywords: multilateral market, syndicate, cooperative game, assignment market, bankruptcy problem, nucleolus

JEL Code: C71, D40
\end{abstract}

\section{Introduction}

The economics of syndicates

A syndicate is a coalition of agents who agree to operate as a single entity. Standard approaches in cooperative game theory do not always yield results in keeping with the idea that syndication in markets is unequivocally profitable. Aumann (1973) considers an exchange economy (or market) with an atomic trader plus a continuum of non-homogeneous traders. He provides examples in which the former is worse off in any core allocation than in the unique competitive one. Thus, the atomic trader would do better by dissolving herself into a continuum of competing traders. Postlewaite and

${ }^{*}$ Corresponding author. E-mail address: toriol@ethz.ch 
Rosenthal (1974) look at a class of markets with two complementary commodities and a finite number of agents, using the core as the set of predicted outcomes. They show in an example that by acting as a syndicate, all agents owning the same commodity can never do better than when they are not syndicated. Legros (1987) extends the analysis of Postlewaite and Rosenthal (1974) to this entire class of markets, employing the nucleolus of the associated cooperative game (Schmeidler, 1969). He shows that whether a syndicate of all agents owning one commodity is advantageous or not depends on the size of its combined endowment relative to that of all agents owning the other commodity. Alternative cooperative solution concepts such as the Shapley value (Gardner, 1977; Guesnerie, 1977; Segal, 2003) also yield the result that syndication can be advantageous in some circumstances and disadvantageous in others. ${ }^{1}$ Similar conclusions can be drawn from non-cooperative approaches to the problem (Okuno et al., 1980; Salant et al., 1983; Bloch and Ghosal, 2000; Fershtman and Gandal, 1994),

Given this failure to obtain clear-cut results, is cooperative game theory an appropriate framework in which to analyze syndication? Aumann (1973) concludes that the core fails in this sense. Postlewaite and Rosenthal (1974), in contrast, argue that syndication might not have any compelling advantages in certain economically meaningful settings. The fact that it is thus not rare for syndicates to be disadvantageous to their members partially rehabilitates the core in their view. Some empirical papers (see e.g. Asch and Seneca, 1976) find evidence that syndication is indeed sometimes associated with lower profitability for firms. The conjecture that antitrust activity always results in lower prices - and higher consumer surplus - has also been disputed empirically (see e.g. Sproul, 1993). All these findings, theoretical and empirical alike, reinforce the need for a greater understanding of the role and potential advantages of syndicates, both positively and normatively. This is the general object of our paper.

\section{Multi-sided assignment markets}

Bilateral assignment markets have come under close scrutiny since the seminal paper of Shapley and Shubik (1972) (one should not forget Gale, 1960). In such markets any gains from trade arise from transactions between a pair of agents, say, a seller and a buyer. In a multi-sided assignment market (Quint, 1991), which includes the bilateral case, there are several types of agents and a positive benefit can only be achieved by forming coalitions that consist of exactly one individual of each type. An instance of a three-sided market is provided by Stuart Jr (1997). He considers an economy where the outcome in one market may affect outcomes in another: a supplier, a firm, and a buyer - as a final consumer - may be needed to carry out a transaction in this setting. Sherstyuk (1999) provides related examples: a future home owner, an architect, and a worker are needed to build and sell a house; a capitalist, an entrepreneur, and a worker are necessary to start a new firm.

It is well-known that when the market comprises at least three types of agents, the core of the associated market game can be empty (Kaneko and Wooders, 1982). The potential lack of stability of this broad family of markets does not mean they are uninteresting. In fact, there exist several classes of multi-sided assignment markets that have a clear-cut economic interpretation and whose

\footnotetext{
${ }^{1}$ We refer to Schmalensee (2007) for the vast industrial organization literature that deals with the incentives for the creation and/or destruction of syndicates.
} 
associated market games have a non-empty core. We shall give additional reasons based on real-world observations why studying such markets is of interest.

IT firms occupy a prominent position in economies nowadays. These firms specialize in software (Microsoft, Google, or Facebook), hardware (Samsung, LG, or Foxconn), or both (IBM, Cisco, or Apple). A three-sided assignment market arises naturally when we consider consumers of technological gadgets that combine hardware and software (an operating system), as these commodities are perfect complements. $^{2}$

When any buyer values each bundle of the component goods equally, the assignment market is called a multi-sided Böhm-Bawerk assignment market (Tejada and Núñez, 2012; Tejada, 2013). Bilateral markets of this type have been extensively analyzed in the literature (von Böhm-Bawerk, 1930; Shapley and Shubik, 1972; Mendelson, 1982; Granot and Granot, 1992; Núñez and Rafels, 2005). While some segments of IT markets feature differentiated products, others consist of products which are indistinguishable to consumers - consider, e.g., laptops of a given size and processor manufactured by Asus and Lenovo. IT three-sided assignment markets with homogeneous products indeed exist and are even common.

\section{Syndication in multi-sided assignment markets}

Two salient types of syndication can be considered for arbitrary multi-sided assignment markets. First, sellers owning the same commodity may collude, giving rise to a horizontal syndicate. This is the type of syndication considered in most of the papers already mentioned. Second, agents owning different commodities may merge and form a vertical syndicate. To date, theoretical understanding of this type of syndication is still limited. The goal of this paper is to make further progress in this direction.

To elaborate, we concentrate on vectors of prices at which the formation of vertical syndicates does not favor any market participant. To be precise, we define a price to be vertical syndicationproof if any buyer's surplus is unaffected when either (a) sellers of different commodities collude into vertical syndicates, or (b) single firms split into more sellers of different commodities. Thus, at a vertical syndication-proof price, vertical syndicates leave the buyers neither worse nor better off. If a vertical syndication-proof price is also competitive, there are no incentives for individual sellers to split artificially or for sellers owning different commodities to collude and form a vertical syndicate.

Several widely known IT firms have faced antitrust regulations in recent years. Microsoft has been sued several times for abusing its market power in the operating system industry. ${ }^{3}$ Google is currently being investigated by the EU for manipulating web search results to favor its own shopping service. ${ }^{4}$ Both examples indicate that public authorities grow concerned when a firm reaches a dominant position in a single market. This fact notwithstanding, antitrust indictments have also targeted acquisitions affecting two vertically related markets. A notorious case is the purchase of Motorola by Google, which involved a firm producing software and a firm producing hardware. ${ }^{5}$ That is,

\footnotetext{
${ }^{2}$ The assumption that technology markets have only three sectors is a defensible simplification, despite the fact that intermediary firms also participate in them.

${ }^{3}$ Economides (2001) analyzes the case of "US against Microsoft".

${ }^{4}$ See http://www.reuters.com/article/2015/04/27/us-eu-google-antitrust-idUSKBNONF1YX20150427, retrieved on 3-July-2015.

${ }^{5}$ See http://www.bbc.com/news/business-18164190, retrieved on 29-June-2015.
} 
syndicates between firms producing different commodities have been formed, and their formation or dissolution is certainly a matter that concerns firms, consumers, and antitrust authorities.

\section{The main result}

Our main finding, viz. Theorem II, is a characterization of the nucleolus of multi-sided BöhmBawerk assignment games using four properties. ${ }^{6}$ To the best of our knowledge, we are the first to provide an axiomatic analysis of such solution concept for a class of market games. More specifically, we show that the nucleolus payoffs yield the unique vector of prices with the following properties: (i) they are vertical syndication-proof, (ii) they are competitive, (iii) they yield the average of the buyers- and the sellers-optimal allocations in bilateral markets, and (iv) they are anonymous with respect to the traders' bargaining power.

The requirement that the labels of market traders be irrelevant, in particular, has already been considered in axiomatic analyses of markets - see e.g. Dubey et al. (1980). More generally, the widely applied two-player Nash bargaining solution (Nash Jr, 1950) treats both agents anonymously when both the set of feasible allocations and the disagreement point are symmetric. The anonymity property that we impose in this paper states that the surplus obtained by each market participant should depend only on her bargaining power and the overall market surplus. ${ }^{7}$

We offer two alternative interpretations of the concept of a trader's bargaining power for the class of markets considered here. First, it can be understood as a proxy for the value she adds to the rest of the market (Brandenburger and Stuart, 2007). In our model, a trader's bargaining power sets an upper bound on her marginal contribution to the market game. Second, the process of Schumpeterian creative destruction exhibits high frequency in the IT sector. This either renders some goods quickly obsolete or drastically reduces the costs of producing them. Innovating firms can appropriate temporarily the monopoly-type returns from investment through either lower costs or higher prices. A firm's bargaining power can then be seen as a measure of the innovation embodied in its product relative to the competition. Similarly, a consumer's bargaining power is a measure of her willingness-to-pay for the bundle in question compared to that of other buyers.

Our analysis also establishes conditions under which vertical syndication-proofness is incompatible with horizontal syndication-proofness. Since a majority of the horizontal syndicates analyzed by antitrust agencies occur in vertically related industries (Milliou and Petrakis, 2007), the analysis of this latter type of syndication is also relevant for the class of markets analyzed in this paper.

\section{A link to bankruptcy}

The proof of our characterization result uses a link that we have discovered between our class of market games and bankruptcy games. Bankruptcy problems involve situations in which several individuals have claims over an estate, but the estate is not sufficient to satisfy all claims. The cooperative approach to this problem was first addressed by O'Neill (1982), and a large literature has followed. Bankruptcy problems are useful in economic theory due to their simplicity and because they embody situations other than bankruptcy, e.g. taxation, cost sharing, or surplus sharing. ${ }^{8}$ Our

\footnotetext{
${ }^{6}$ We do not claim that the nucleolus and the properties characterizing are the only reasonable possibilities.

${ }^{7}$ The impact of buyers' bargaining power on their surplus was analyzed in Inderst and Wey (2007).

${ }^{8}$ See Thomson $(2003,2015)$ for an extensive survey of the subject.
} 
paper complements this literature in that we identify a subclass of market games that is isomorphic to the entire class of bankruptcy games - see Theorem I. The insights provided by this isomorphism enable us to translate rules, properties, and results from one setting to another. Special attention is paid to how the Talmud Bankruptcy Rule (Aumann and Maschler, 1985) translates into our class of markets. Our findings thus reinforce the relevance and applicability of the game-theoretical model of the bankruptcy problem.

\section{Organization of the paper}

The paper is organized as follows. In Section 2 we present the main definitions and illustrate them by examples. In Section 3 we establish a link between multi-sided Böhm-Bawerk assignment games and bankruptcy games. In Section 4 we propose a procedure to define (payoff) rules in our class of markets from rules in bankruptcy problems, and vice versa. In Section 5 we analyze different properties that rules in both settings might satisfy. In Section 6 we build on the previous sections to cast light on the relation between the two aforementioned groups of properties. This helps us characterize the unique rule in our class of markets that yields a vertical syndication-proof vector of competitive prices and which satisfies three additional properties. In Section 7 we analyze alternative forms of syndication. Section 8 concludes. The proofs of all auxiliary results can be found in the Appendix.

\section{Notation and preliminaries}

A cooperative game with transferable utility (in short, a game) is a pair $(N, v)$, where $N$ is a finite set of players and $v$, the characteristic function, is a real-valued function on $2^{N}=\{S: S \subseteq N\}$, with $v(\emptyset)=0$. We denote by $\mathcal{G}$ the set of all games. For every finite set $N,\left(N, v_{0}\right)$ stands for the null game, where for every $S \subseteq N, v_{0}(S)=0$. The set of non-negative real numbers is denoted by $\mathbb{R}_{+}$, and for every $x \in \mathbb{R}$ we use the notation $(x)_{+}=\max \{0, x\}$. We denote by $\mathbb{R}^{N}$ the $|N|$-dimensional Euclidean space with elements $x \in \mathbb{R}^{N}$ having components $x_{i}, i \in N$. A vector $x \in \mathbb{R}^{N}$ is called an allocation or payoff vector. For $S \subseteq N$ and $x \in \mathbb{R}^{N}$, let as usual $x(S)=\sum_{i \in S} x_{i}$. We say that $x \in \mathbb{R}^{N}$ is an imputation of $(N, v)$ if $x(N)=v(N)$ and $x_{i} \geq v(\{i\})$ for all $i \in N$. We let $I(N, v)$ denote the set of imputations of $(N, v)$. The core of a game is the set of efficient allocations that cannot be improved upon by any coalition on its own, i.e.,

$$
C(N, v)=\left\{x \in \mathbb{R}^{N}: x(N)=v(N) \text { and for every } S \subsetneq N, x(S) \geq v(S)\right\} .
$$

Lastly, for $x \in I(N, v)$ and $S \in 2^{N} \backslash\{\emptyset, N\}$, the excess of coalition $S$ at $x$ is defined by $e_{v}(S, x)=$ $v(S)-x(S)$, and it is a measure of the satisfaction of coalition $S$ with respect to allocation $x$. Given $(N, v) \in \mathcal{G}$ with $I(N, v) \neq \emptyset$ and $x \in I(N, v)$, we define $\lambda(x) \in \mathbb{R}^{2^{N} \backslash\{\emptyset, N\}}$ as the vector of excesses of all proper coalitions of $N$ arranged in a non-increasing order. That is, $\lambda_{k}(x)=e_{v}\left(S_{k}, x\right)$ for all $k \in\left\{1, \ldots, 2^{n}-2\right\}$ and $\lambda_{k}(x) \geq \lambda_{j}(x)$ if $1 \leq k<j \leq 2^{n}-2$, where $\left\{S_{1}, \ldots, S_{2^{n}-2}\right\}$ is the set of all proper coalitions of $N$. The nucleolus of $(N, v)$ is the imputation $\eta(N, v)$ that minimizes $\lambda(x)$ with respect to the lexicographic order over the set of imputations. That is, $\lambda(\eta(v)) \leq_{\text {Lex }} \lambda(x)$ for all 
$x \in I(N, v) \cdot{ }^{9}$ It is well known that the nucleolus is always a single point and whenever the core of the game is non-empty, it is a core allocation.

\subsection{Böhm-Bawerk assignment markets and games}

In this section, we introduce a particular class of multilateral assignment markets and we associate a game with each of these markets.

Definition 2.1. Given an integer $m>1$, an $m$-sided Böhm-Bawerk assignment market (in short, a market) is a tuple $\left(N^{1}, \ldots, N^{m}, \mathbf{c}, w\right)$, where $N^{1}, \ldots, N^{m-1}$ are the finite sets of sellers, $N^{m}$ is the finite set of buyers, $\mathbf{c}=\left(c^{1}, \ldots, c^{m-1}\right) \in \mathbb{R}_{+}^{N^{1}} \times \cdots \times \mathbb{R}_{+}^{N^{m-1}}$, and $w \in \mathbb{R}_{+}^{N^{m}}$.

The $m$ sets of agents $N^{1}, \ldots, N^{m}$ are called sectors. Unless stated to the contrary, henceforth we let $M=\{1, \ldots, m\}$ denote the set of sectors. Each seller owns exactly one unit of an indivisible commodity. Each buyer is willing to buy a bundle composed of exactly one unit of each commodity. The goods owned by two sellers belong to the same commodity if and only if both sellers belong to the same sector. Hence in an $m$-sided market there are $m-1$ different commodities. For each $k \in M \backslash\{m\}, c_{i}^{k}$ stands for the valuation of seller $i \in N^{k}$ of her own good (or simply the cost). To buyer $i \in N^{m}, w_{i}$ stands for her valuation of a lot consisting of one good of each of the commodities. Without loss of generality, we assume that $\left|N^{1}\right|=\cdots=\left|N^{m}\right|=n$ by introducing dummy sellers with very large reservation prices and dummy buyers with zero willingness-to-pay. We arrange sellers' valuations in a non-decreasing order, and buyers' valuations in a non-increasing order. That is, for every $k \in M \backslash\{m\}, c_{1}^{k} \leq \cdots \leq c_{n}^{k}$ and $w_{1} \geq \cdots \geq w_{n} \cdot{ }^{10}$ Let $\mathcal{B B M}$ stand for the set of all such markets. When no confusion regarding the set of agents can arise, we will denote a market simply by $(\mathbf{c}, w)$.

Next, we present an example that will be used throughout the paper to illustrate certain definitions.

Example 2.1. Consider the 24-person three-sided market given by

$$
\begin{aligned}
\left(c_{1}, c_{2}, w\right)= & (30,33,40,48,50,55,70,78 ; \\
& 25,35,58,59,60,67,70,82 ; \\
& 200,180,160,140,130,120,100,90) .
\end{aligned}
$$

We abuse language and henceforth use tuple indistinctly to refer both to the $m$-tuple consisting of one agent of each type $Z \in \prod_{k=1}^{m} N^{k}$ as well as to the coalition composed of all agents in the $m$-tuple. A matching among $N^{1}, \ldots, N^{m}$ is a set of $n$ pairwise disjoint tuples. We denote by $\mathcal{M}\left(N^{1}, \ldots, N^{m}\right)$ the set of all matchings among $N^{1}, \ldots, N^{m}$. Given $(\mathbf{c}, w) \in \mathcal{B B M}$, the mapping $a(\mathbf{c}, w): \prod_{k=1}^{m} N^{k} \rightarrow \mathbb{R}_{+}$describes the potential benefit obtained by every tuple. That is, for $Z=\left(i_{1}, \ldots, i_{m}\right) \in \prod_{k=1}^{m} N^{k}$

$$
a(\mathbf{c}, w)(Z)=\left(w_{i_{m}}-\sum_{k \in M \backslash\{m\}} c_{i_{k}}^{k}\right)_{+} .
$$

\footnotetext{
${ }^{9}$ Given $x, y \in \mathbb{R}^{n}$, we say $x<_{\text {Lex }} y$ if there is some $1 \leq i \leq n$ such that $x_{i}<y_{i}$ and $x_{j}=y_{j}$ for $1 \leq j<i$. Also, we say $x \leq_{\text {Lex }} y$ if $x<_{\text {Lex }} y$ or $x=y$.

${ }^{10}$ This assumption enables us to speak properly about the $i^{\text {th }}$ agent of sector $k \in M$.
} 
When no confusion can arise, we will denote $a(\mathbf{c}, w)(Z)$ simply by $a_{Z}$.

If money exists in the market and side payments between agents are allowed, a market $(\mathbf{c}, w)$ generates an assignment game $\left(N, \omega^{\mathbf{c}, w}\right) \in \mathcal{G}$, where $N=\cup_{k=1}^{m} N^{k}$ and for every $S \subseteq N$,

$$
\omega^{\mathbf{c}, w}(S)=\max _{\mu \in \mathcal{M}\left(N^{1} \cap S, \ldots, N^{m} \cap S\right)}\left\{\sum_{Z \in \mu} a_{Z}\right\},
$$

and the summation over the empty set is zero. That is, the assignment game describes the best outcome that players in each coalition can achieve by organizing themselves in tuples. Hence, it is a tool for the analysis of the division of the net profit $\omega^{\mathbf{c}, w}(N)$ between buyers and sellers. We denote by $\mathcal{B B G}$ the set of all assignment games associated with markets in $\mathcal{B B M}$.

Tejada (2013) studies the core of an arbitrary game in $\mathcal{B B G}$ by means of the so-called sectors game, in which the (fictitious) players are the sectors of the market. Below we recall the main result therein, but first we introduce some concepts to facilitate understanding. For every $i \in \mathbb{N}$, let $Z^{i}=(i, \ldots, i) \in \mathbb{R}^{m}$. Then, for every $(\mathbf{c}, w) \in \mathcal{B B M}$ let

$$
r(\mathbf{c}, w)=\max _{i \in\{1, \ldots, n\}}\left\{i: a_{Z^{i}}>0\right\},
$$

with the convention that $r(\mathbf{c}, w)=0$ if $a(\mathbf{c}, w)$ is the null mapping. When the market is clear from the context, we will denote $r(\mathbf{c}, w)$ simply by $r$.

Example 2.1 (cont.). We can arrange the primitives of the market-note that $r=5$, as indicated by the boldface numbers - as follows:

\begin{tabular}{|c|c|c|c|c|}
\hline Sector 1 & Sector 2 & Sector 3 & Surplus & Truncated surplus \\
\hline$c_{i}^{1}$ & $c_{j}^{2}$ & $w_{k}$ & $w_{k}-c_{i}^{1}-c_{j}^{2}$ & $\left(w_{k}-c_{i}^{1}-c_{j}^{2}\right)_{+}$ \\
\hline 30 & 25 & 200 & 145 & 145 \\
33 & 35 & 180 & 112 & 112 \\
40 & 58 & 160 & 62 & 62 \\
48 & 59 & 140 & 33 & 33 \\
\hline $\mathbf{5 0}$ & $\mathbf{6 0}$ & $\mathbf{1 3 0}$ & $\mathbf{2 0}$ & 20 \\
55 & 67 & 120 & -2 & 0 \\
\hline 70 & 70 & 100 & -40 & 0 \\
78 & 82 & 90 & -70 & 0 \\
\hline
\end{tabular}

For every $S \subseteq M$ we introduce the notation $Z^{S}=r \mathbb{1}_{S}+(r+1) \mathbb{1}_{M \backslash S} \in \mathbb{R}^{m}$, where for every $T \subseteq M, \mathbb{1}_{T} \in \mathbb{R}^{m}$ is the vector such that $\mathbb{1}_{T}(k)=1$ if $k \in T$ and $\mathbb{1}_{T}(k)=0$ if $k \notin T$. Notice that by introducing dummy players, we can always ensure that $r+1<n$, so there are at least $r+2$ agents in all sectors. Also note that $\left\{Z^{S}: S \subseteq M\right\}$ are precisely all the tuples that can be obtained by combining the $r^{\text {th }}$ and $r+1^{\text {th }}$ agents of each sector.

Definition 2.2. Given $(\mathbf{c}, w) \in \mathcal{B B M}$, the associated sectors game $\left(M, v^{\mathbf{c}, w}\right) \in \mathcal{G}$ is the game defined for every $S \subseteq M$ by

$$
v^{\mathbf{c}, w}(S)=\left\{\begin{array}{cc}
a_{Z^{S}} & \text { if } r>0 \\
0 & \text { if } r=0
\end{array}\right.
$$


We denote by $\mathcal{S G}$ the set of all sectors games associated with markets in $\mathcal{B B} \mathcal{M}$.

Example 2.1 (cont.). The sectors game $\left(M, v^{\mathbf{c}, w}\right)$ is given by $M=\{1,2,3\}$ and

$$
\begin{array}{ll}
v^{\mathbf{c}, w}(\{1\})=3, & v^{\mathbf{c}, w}(\{1,2\})=10, \\
v^{\mathbf{c}, w}(\{2\})=5, & v^{\mathbf{c}, w}(\{1,3\})=13, \quad v^{\mathbf{c}, w}(\{1,2,3\})=20 . \\
v^{\mathbf{c}, w}(\{3\})=8, & v^{\mathbf{c}, w}(\{2,3\})=15,
\end{array}
$$

Payoffs in the sectors game belong to a different space than those of the corresponding market game $\left(\mathbb{R}^{M}\right.$ versus $\left.\mathbb{R}^{N^{1}} \times \ldots \times \mathbb{R}^{N^{m}}\right)$. Since the main result in Tejada (2013) relates the core of both games, we next introduce two tools that will allow us to map the payoffs of one space onto the payoffs of another space.

First, for every $(\mathbf{c}, w) \in \mathcal{B B} \mathcal{M}$ we introduce the replica operator, $\mathcal{R}_{\mathbf{c}, w}$, which is an injective linear function defined by

$$
\begin{aligned}
\mathcal{R}_{\mathbf{c}, w}: \mathbb{R}^{M} & \longrightarrow \\
\left(x_{1}, \ldots, x_{m}\right) & \longrightarrow(\overbrace{x_{1}, \ldots, x_{1}}^{\mathbb{R}^{N^{1}}}, \overbrace{0, \ldots, 0}^{\times} ; \quad \ldots \quad ; \overbrace{x_{m}, \ldots, x_{m}}^{n-r}, \overbrace{0, \ldots, 0}^{n_{0}^{n-r}}) .
\end{aligned}
$$

Example 2.1 (cont.). The replica operator is given by

$$
\mathcal{R}_{\mathbf{c}, w}\left(x_{1}, x_{2}, x_{3}\right)=\left(x_{1}, x_{1}, x_{1}, x_{1}, x_{1}, 0,0,0 ; x_{2}, x_{2}, x_{2}, x_{2}, x_{2}, 0,0,0 ; x_{3}, x_{3}, x_{3}, x_{3}, x_{3}, 0,0,0\right) .
$$

Second, for every $(\mathbf{c}, w) \in \mathcal{B B M}$ we introduce the translation vector

$$
t_{\mathbf{c}, w}=\left(t_{1}^{1}, \ldots, t_{n}^{1} ; \ldots ; t_{1}^{m}, \ldots, t_{n}^{m}\right) \in \mathbb{R}^{N^{1}} \times \cdots \times \mathbb{R}^{N^{m}}
$$

which is defined for every $i \in\{1, \ldots, n\}$ by

$$
t_{i}^{k}= \begin{cases}\left(c_{r}^{k}-c_{i}^{k}\right)_{+} & \text {if } k \in M \backslash\{m\} \\ \left(w_{i}-w_{r}\right)_{+} & \text {if } k=m\end{cases}
$$

if $r(\mathbf{c}, w)>0$ and by $t_{\mathbf{c}, w}=(0, \ldots, 0)$ if $r(\mathbf{c}, w)=0$.

Example 2.1 (cont.). The translation vector is given by

$$
t_{\mathbf{c}, w}=(20,17,10,2,0,0,0,0 ; 35,25,2,1,0,0,0,0 ; 70,50,30,10,0,0,0,0) .
$$

Lastly, for every $t \in \mathbb{R}^{l}$ and $B \subseteq \mathbb{R}^{l}$, let $t+B$ denote the translated set $B$ by the vector $t$, i.e., $t+B=\{t+x: x \in B\}$. We are now in a position to state both the main result in Tejada (2013) and the main result in Tejada and Núñez (2012).

Theorem 2.1. (Tejada, 2013; Tejada and Núñez, 2012) For every market $(\mathbf{c}, w) \in \mathcal{B B} \mathcal{M}$,

(i) $C\left(N, \omega^{\mathbf{c}, w}\right)=t_{\mathbf{c}, w}+\mathcal{R}_{\mathbf{c}, w}\left(C\left(M, v^{\mathbf{c}, w}\right)\right) \neq \emptyset$,

(ii) $\eta\left(N, \omega^{\mathbf{c}, w}\right)=t_{\mathbf{c}, w}+\mathcal{R}_{\mathbf{c}, w}\left(\eta\left(M, v^{\mathbf{c}, w}\right)\right)$.

Part $(i)$ indicates that the variability of the core of an assignment game can be entirely explained by the core of the corresponding sectors game. Accordingly, the core of the assignment game associated with the 24-person market in Example 2.1 is determined (up to a bijection) by the core of the 3-player 
sectors game also described in Example 2.1. The interests of all agents that belong to the same sector are thus perfectly aligned in the core. Part (ii) states that the nucleolus of the assignment game can also be obtained by translating the nucleolus of the sectors game.

The only information relevant for the variability of the core of a market is contained in the positions $r$ and $(r+1)$ of the vectors $c^{1}, \ldots, c^{m-1}, w$. In particular, the agents in the $(r+1)^{\text {th }}$ position are important even though they do not contribute to creating any value. The reason is that they determine the best outside option of the agents in the $r^{\text {th }}$ position. To every market $\left(N^{1}, \ldots, N^{m}, \mathbf{c}, w\right) \in \mathcal{B B M}$, we then propose associating another market $\left(\widetilde{N}^{1}, \ldots, \widetilde{N}^{m}, \widetilde{\mathbf{c}}, \widetilde{w}\right)$ where $\left|\tilde{N}^{1}\right|=\ldots=\left|\tilde{N}^{m}\right|=2$ and

$$
(\widetilde{\mathbf{c}}, \widetilde{w})= \begin{cases}\left(\left(c_{r}^{1}, c_{r+1}^{1} ; \ldots ; c_{r}^{m-1}, c_{r+1}^{m-1}\right),\left(w_{r}, w_{r+1}\right)\right) & \text { if } r(\mathbf{c}, w)>0 \\ \left(\left(c_{1}^{1}, c_{2}^{1} ; \ldots ; c_{1}^{m}, c_{2}^{m}\right),\left(w_{1}, w_{2}\right)\right) & \text { if } r(\mathbf{c}, w)=0\end{cases}
$$

It is straightforward to check that $\left(M, v^{\mathbf{c}, w}\right)=\left(M, v^{\tilde{\mathbf{c}}, \tilde{w}}\right)$.

In the following definition, we formally introduce the class of all markets obtained from markets in $\mathcal{B B M}$ by the above procedure. These markets will play an important role throughout the paper.

Definition 2.3. Given an integer $m>1$, a 2-regular market is a market $\left(N^{1}, \ldots, N^{m}, \mathbf{c}, w\right) \in \mathcal{B B M}$ such that $\left|N^{1}\right|=\cdots=\left|N^{m}\right|=2$ and

$$
w_{2}-\sum_{k \in M \backslash\{m\}} c_{2}^{k} \leq 0
$$

We denote by $2-\mathcal{B B M}$ the set of all 2-regular markets and by $2-\mathcal{B B G}$ the set of assignment games associated with 2-regular markets. Note that in a 2-regular market, the only possible transaction takes place between the agents in the first position of each sector. Hence, we say that these agents are active, while the agents in the second position are inactive. For every $(\mathbf{c}, w) \in \mathcal{Q}-\mathcal{B B M}$ and $k \in M$, we define the bargaining power of the active agent of sector $k$ when $r(\mathbf{c}, w)=1$ as

$$
b^{k}(\mathbf{c}, w)=\left\{\begin{array}{cl}
c_{2}^{k}-c_{1}^{k} & \text { if } k \in M \backslash\{m\}, \\
w_{1}-w_{2} & \text { if } k=m .
\end{array}\right.
$$

The bargaining power of active agents when $r(\mathbf{c}, w)=0$ and of inactive agents is zero by definition. We slightly abuse language and refer to $b^{k}(\mathbf{c}, w)$ as the bargaining power of sector $k .^{11}$

The following two remarks are straightforward:

Remark 2.1. For every 2-regular market, the marginal contribution of every agent to the grand coalition in the market game is bounded from above by her bargaining power. ${ }^{12}$

Remark 2.2. The set of sector games associated with 2-regular markets is $\mathcal{S G}$.

\footnotetext{
${ }^{11}$ For convenience, we define the bargaining power only in 2-regular markets. However, the definition can be generalized to arbitrary markets in $\mathcal{B B M}$ by using Eq. (2) and the translation vector.

${ }^{12}$ Given a game $(N, v)$ and a player $i \in N$, the marginal contribution of $i$ to the grand coalition is the amount $v(N)-v(N \backslash\{i\})$.
} 


\subsection{Bankruptcy problems and games}

In this section, we formally introduce bankruptcy problems and bankruptcy games.

Definition 2.4. Given an integer $m>1$, a bankruptcy problem is a tuple $(M, E, d)$, where $M=$ $\{1, \ldots m\}$ is the finite set of agents, $E \geq 0$ is the estate to be divided, and $d \in \mathbb{R}_{+}^{M}$ is the vector of claims, which satisfy $\sum_{i \in M} d_{i} \geq E$.

We denote by $\mathcal{B P}$ the set of all bankruptcy problems. When no confusion can arise, we will omit the set of players and write $(E, d) \in \mathcal{B P}$. Given a bankruptcy problem $(E, d) \in \mathcal{B P}$, the associated bankruptcy game $\left(M, v^{E, d}\right) \in \mathcal{G}$ is defined for every $S \subseteq M$ by

$$
v^{E, d}(S)=(E-d(M \backslash S))_{+} .
$$

The worth of a coalition according to the bankruptcy game is the amount that is left after all other claims have been satisfied, assuming there is something left over. We denote by $\mathcal{B G}$ the set of all bankruptcy games obtained from bankruptcy problems. Let us illustrate the definition with the example below.

Example 2.2. Consider the bankruptcy problem where $M=\{1,2,3\}, E=20$, and $d=(5,7,10)$. The associated bankruptcy game $\left(M, v^{E, d}\right)$ is then given by:

$$
\begin{array}{ll}
v^{E, d}(\{1\})=3, & v^{E, d}(\{1,2\})=10, \\
v^{E, d}(\{2\})=5, & v^{E, d}(\{1,3\})=13, \quad v^{E, d}(\{1,2,3\})=20 . \\
v^{E, d}(\{3\})=8, & v^{E, d}(\{2,3\})=15,
\end{array}
$$

\section{Connection between assignment and bankruptcy games}

In this section, we show that there exists a link between bankruptcy problems and markets.

Theorem I. The set of bankruptcy games and the set of sector games associated with markets coincide, i.e.,

$$
\mathcal{B G}=\mathcal{S G}
$$

Proof. On the one hand, for every $(M, E, d) \in \mathcal{B P}$ with $M=\{1, \ldots, m\}$, we define a market, $(\mathbf{c}(E, d), w(E, d)) \in \mathcal{B B M}$, with two agents in each sector, i.e., $\mathbf{c}(E, d) \in \mathbb{R}_{+}^{2(m-1)}$ and $w(E, d) \in \mathbb{R}_{+}^{2}$, as follows:

$$
\begin{aligned}
\mathbf{c}(E, d) & =\left(\frac{d_{m}}{m-1}, d_{1}+\frac{d_{m}}{m-1} ; \ldots ; \frac{d_{m}}{m-1}, d_{m-1}+\frac{d_{m}}{m-1}\right), \\
w(E, d) & =\left(E+d_{m}, E\right)
\end{aligned}
$$

From the definition of the bankruptcy problem, it follows that $(\mathbf{c}(E, d), w(E, d)) \in 2-\mathcal{B B} \mathcal{M}$. Indeed,

$$
w_{2}(E, d)-\sum_{k \in M \backslash\{m\}} c_{2}^{k}(E, d)=E-\sum_{k \in M \backslash\{m\}}\left(d_{k}+\frac{d_{m}}{m-1}\right)=E-\sum_{k \in M} d_{k} \leq 0 .
$$

Note that the exact identity of the players in $(E, d)$ is important, as the valuations of the buyers in $(\mathbf{c}(E, d), w(E, d))$ are associated with $E$ and the claim of the agent $m$ in $(E, d)$. The sectors game 
associated with $(\mathbf{c}(E, d), w(E, d))$ is then defined for every $S \subseteq M \backslash\{m\}$ by

$$
\begin{aligned}
v^{\mathbf{c}(E, d), w(E, d)}(S) & =\left(E-\sum_{k \in S} \frac{d_{m}}{m-1}-\sum_{k \in M \backslash(S \cup\{m\})}\left[d_{k}+\frac{d_{m}}{m-1}\right]\right)_{+} \\
& =(E-d(M \backslash S))_{+}=v^{E, d}(S),
\end{aligned}
$$

and

$$
\begin{aligned}
v^{\mathbf{c}(E, d), w(E, d)}(S \cup\{m\}) & =\left(E+d_{m}-\sum_{k \in S} \frac{d_{m}}{m-1}-\sum_{k \in M \backslash(S \cup\{m\})}\left[d_{k}+\frac{d_{m}}{m-1}\right]\right)_{+} \\
& =(E-d(M \backslash(S \cup\{m\})))_{+}=v^{E, d}(S \cup\{m\}) .
\end{aligned}
$$

On the other hand, for every $\left(N^{1}, \ldots, N^{m}, \mathbf{c}, w\right) \in 2-\mathcal{B B M}$ we define a bankruptcy problem $(E(\mathbf{c}, w), d(\mathbf{c}, w))$, where $E(\mathbf{c}, w) \in \mathbb{R}_{+}$and $d(\mathbf{c}, w) \in \mathbb{R}_{+}^{m}$ are as follows:

$$
\begin{aligned}
& E(\mathbf{c}, w)=\left(w_{1}-\sum_{k \in M \backslash\{m\}} c_{1}^{k}\right)_{+}, \\
& d(\mathbf{c}, w)=\left(c_{2}^{1}-c_{1}^{1}, \ldots, c_{2}^{m-1}-c_{1}^{m-1}, w_{1}-w_{2}\right) .
\end{aligned}
$$

Again, note that the agent $m$ in $(M, E(\mathbf{c}, w), d(\mathbf{c}, w))$ is always associated with the buyers' sectors in $(\mathbf{c}, w)$. It immediately follows that $(E(\mathbf{c}, w), d(\mathbf{c}, w)) \in \mathcal{B P}$. Indeed,

$$
\begin{aligned}
\sum_{k \in M} d_{k}(\mathbf{c}, w) & =\sum_{k \in M \backslash\{m\}}\left(c_{2}^{k}-c_{1}^{k}\right)+\left(w_{1}-w_{2}\right)=\left(w_{1}-\sum_{k \in M \backslash\{m\}} c_{1}^{k}\right)-\left(w_{2}-\sum_{k \in M \backslash\{m\}} c_{2}^{k}\right) \\
& \geq w_{1}-\sum_{k \in M \backslash\{m\}} c_{1}^{k},
\end{aligned}
$$

where the inequality holds since $(\mathbf{c}, w) \in 2-\mathcal{B B M}$. Since $\sum_{k \in M} d_{k}(\mathbf{c}, w) \geq 0$, we conclude that $\sum_{k \in M} d_{k}(\mathbf{c}, w) \geq\left(w_{1}-\sum_{k \in M \backslash\{m\}} c_{1}^{k}\right)_{+}=E(\mathbf{c}, w)$. The associated bankruptcy game is then defined for every $S \subseteq M \backslash\{m\}$ by

$$
\begin{aligned}
v^{E(\mathbf{c}, w), d(\mathbf{c}, w)}(S) & =\left(\left(w_{1}-\sum_{k \in M \backslash\{m\}} c_{1}^{k}\right)_{+}-\left(w_{1}-w_{2}\right)-\sum_{k \in M \backslash(S \cup\{m\})}\left(c_{2}^{k}-c_{1}^{k}\right)\right)+ \\
& =\left(w_{2}-\sum_{k \in S} c_{1}^{k}-\sum_{k \in M \backslash(S \cup\{m\})} c_{2}^{k}\right)_{+}=v^{\mathbf{c}, w}(S)
\end{aligned}
$$

and

$$
\begin{aligned}
v^{E(\mathbf{c}, w), d(\mathbf{c}, w)}(S \cup\{m\}) & =\left(\left(w_{1}-\sum_{k \in M \backslash\{m\}} c_{1}^{k}\right)_{+}-\sum_{k \in M \backslash(S \cup\{m\})}\left(c_{2}^{k}-c_{1}^{k}\right)\right) \\
& =\left(w_{1}-\sum_{k \in S} c_{1}^{k}-\sum_{k \in M \backslash(S \cup\{m\})} c_{2}^{k}\right)_{+}=v^{\mathbf{c}, w}(S \cup\{m\}) .
\end{aligned}
$$


Several comments are in order. First, the above theorem shows that for every game obtained from a bankruptcy problem there exists a market whose associated sectors game is the former game, and vice versa. For instance, the bankruptcy game of Example 2.2 corresponds to the sectors game associated with the 2-regular market of Example 2.1.

Second, it can be verified that the mapping defined by Eq. (4) is injective but not surjective, and that the mapping defined by Eq. (5) is surjective but not injective. Consequently, for each bankruptcy problem, there exists a 2-regular market such that the former can be obtained from the latter via Eq. (5). However, there are 2-regular markets such that there does not exist any bankruptcy problem which can be transformed via Eq. (4) into the former.

Third, together with part ( $i$ ) of Theorem 2.1, we have shown that the variability of the core of an arbitrary multi-sided Böhm-Bawerk assignment game is described by the core of a bankruptcy game, and vice versa. This latter finding raises the question whether a more general class of market games could also be linked to the more general class of convex games. This is left to further research.

Fourth and last, a consequence of Theorem I - see also Proposition 8.1 in the Appendix - is that the entire class of bankruptcy games can be seen as a subclass of assignment games.

Corollary 3.1. The set of assignment games associated to 2-regular markets, 2-BBG, and the set of bankruptcy games, $\mathcal{B G}$, are isomorphic, i.e.,

$$
\mathcal{B G}=\mathcal{S G} \cong 2-\mathcal{B B G} \subseteq \mathcal{B B G}
$$

\section{Exploiting the link to define rules}

In this section, we exploit the insights revealed by the connection between markets and bankruptcy problems, and focus on point-valued solution concepts (or rules). Given $\mathcal{H} \subseteq \mathcal{B B M}$, a rule in $\mathcal{H}$ is a map, $\mathrm{f}$, that associates with every $\left(N^{1}, \ldots N^{m}, \mathbf{c}, w\right) \in \mathcal{H}$, a payoff vector $\mathrm{f}(\mathbf{c}, w)=\left(\mathrm{f}^{k}(\mathbf{c}, w)\right)_{k \in M}$,

where for every $k \in M, \mathrm{f}^{k}(\mathbf{c}, w)=\left(\mathrm{f}_{i}^{k}(\mathbf{c}, w)\right)_{i \in N^{k}} \in \mathbb{R}^{N^{k}}$. A rule in $\mathcal{B P}$ is a map, fb, that associates with every $(M, E, d) \in \mathcal{B P}$ a payoff vector $\mathrm{fb}(E, d)=\left(\mathrm{fb}_{i}(E, d)\right)_{i \in M} \in \mathbb{R}^{M}$.

Theorem 2.1 together with Theorem I and its proof suggest a natural procedure to define rules in $\mathcal{B B M}$ based on rules in $\mathcal{B P}$, and vice versa. ${ }^{13}$ On the one hand, for every rule fb in $\mathcal{B P}$, we define a rule $\Phi(\mathrm{fb})$ in $\mathcal{B B M}$ constructed for every market $(\mathbf{c}, w) \in \mathcal{B B M}$ according to the following steps:

1. Define the market $(\widetilde{\mathbf{c}}, \widetilde{w})$ from $(\mathbf{c}, w)$ by means of Eq. (2).

2. Define the bankruptcy problem $(E(\widetilde{\mathbf{c}}, \widetilde{w}), d(\widetilde{\mathbf{c}}, \widetilde{w}))$ from $(\widetilde{\mathbf{c}}, \widetilde{w})$ by means of Eq. (5).

3. Apply fb to the bankruptcy problem to obtain an allocation.

4. Translate the allocation of the bankruptcy problem to the original market by means of the transformation used in Theorem 2.1.

\footnotetext{
${ }^{13}$ Theorem 2.1 is concerned with the core of the assignment game. Consequently, the translations that we present here are especially applicable when the rule that is being translated proposes core allocations. However, to study how properties in one setting are translated to the other setting, it is convenient to be able to translate any rule in $\mathcal{B P}$ into a rule in $\mathcal{B B M}$, and vice versa.
} 
Formally, we have defined a mapping $\Phi$ that assigns a rule in $\mathcal{B B M}$ to every rule fb in $\mathcal{B P}$ as follows:

$$
\Phi(\mathrm{fb})(\mathbf{c}, w)=t_{\mathbf{c}, w}+\mathcal{R}_{\mathbf{c}, w}(\mathrm{fb}(E(\widetilde{\mathbf{c}}, \widetilde{w}), d(\widetilde{\mathbf{c}}, \widetilde{w}))) .
$$

On the other hand, for every rule $f$ in $\mathcal{B B M}$ we conversely define a rule $\Psi(f)$ in $\mathcal{B P}$ constructed for every bankruptcy problem $(E, d) \in \mathcal{B P}$ according to the following steps:

1. Define the 2-regular market $(\mathbf{c}(E, d), w(E, d))$ from $(E, d)$ by means of Eq. (4).

2. Apply $f$ to the 2-regular market to obtain an allocation.

3. Translate the allocation of the market to the original bankruptcy problem by taking only the payoffs to the agents in the first position in each sector of the market.

Formally, we have defined a mapping $\Psi$ that assigns a rule in $\mathcal{B P}$ to every rule $f$ in $\mathcal{B B M}$ as follows:

$$
\Psi(\mathrm{f})(E, d)=\left(\mathrm{f}_{1}^{k}(\mathbf{c}(E, d), w(E, d))\right)_{k \in M} .
$$

\section{Properties of rules}

In this section, we consider several properties that both rules in $\mathcal{B B M}$ and in $\mathcal{B P}$ might satisfy. We start with some well-known properties for rules in $\mathcal{B P}$, and then we propose some new properties for rules in $\mathcal{B B M}$.

\section{$5.1 \quad$ Bankruptcy problems}

The literature regarding properties of rules in $\mathcal{B P}$ is vast. Here, we consider four of them.

WD A rule in $\mathcal{B P}, \mathrm{fb}$, is well-defined if for every $(E, d) \in \mathcal{B P},{ }^{14}$

$$
0 \leq \mathrm{f}_{i}(E, d) \leq d_{i} \quad \text { and } \quad \sum_{i \in M} \mathrm{f}_{i}(E, d)=E .
$$

The above property requires very mild assumptions, i.e., that all the estate is divided among all the players and that none of them receives more than her claim.

CO A rule in $\mathcal{B P}, \mathrm{fb}$, satisfies consistency if for every $(M, E, d) \in \mathcal{B P}$ and $S \subseteq M$, if we set $x=$ $\mathrm{fb}(M, E, d)$, then

$$
x_{S}=\mathrm{fb}\left(S, x(S), d_{S}\right) .
$$

The above property requires that the payoffs remain unaffected if some agents leave the problem with the payoffs proposed by the rule, and they bargain again for the division of the joint payoff with reference to the original claims.

\footnotetext{
${ }^{14}$ This condition is usually imposed on the definition of a rule. However, to compare rules and properties of bankruptcy problems and markets, it is better to consider WD as a property of a rule in $\mathcal{B P}$. In particular, note that the translation $\Psi$ may give rise to a rule in $\mathcal{B P}$ that is not well defined.
} 
$\mathrm{BC}$ A rule in $\mathcal{B P}$, fb, satisfies bilateral consistency if for every $(M, E, d) \in \mathcal{B P}$ and $S \subseteq M$ such that $|S|=2$, if we set $x=\mathrm{fb}(M, E, d)$, then

$$
x_{S}=\mathrm{fb}\left(S, x(S), d_{S}\right) .
$$

The above property requires that consistency holds only for subsets of two players.

CG A rule in $\mathcal{B P}, \mathrm{fb}$, satisfies the contested garment property if for every $(M, E, d) \in \mathcal{B P}$ with $M=\{1,2\}$,

$$
\mathrm{fb}(E, d)=\left(\frac{E+\left(E-d_{2}\right)_{+}-\left(E-d_{1}\right)_{+}}{2}, \frac{E+\left(E-d_{1}\right)_{+}-\left(E-d_{2}\right)_{+}}{2}\right) .
$$

The above property proposes a particular division of the estate for the two-claimant case based on the concede-and-divide principle (Dagan, 1996; Moreno-Ternero and Villar, 2006).

Aumann and Maschler (1985) show that the properties listed above lead to a unique possibility.

Theorem 5.1. (Aumann and Maschler, 1985) There exists a unique rule in $\mathcal{B P}$, the so-called Talmud Bankruptcy Rule, that satisfies WD, BC, and CG. Moreover, for every bankruptcy problem this rule chooses the nucleolus of the associated bankruptcy game.

Two comments are in order. First, the Talmud Bankruptcy Rule, henceforth Tb, admits for every $(M, E, d) \in \mathcal{B P}$ and $k \in M$ the following expression:

$$
\operatorname{Tb}_{k}(M, E, d)= \begin{cases}\min \left\{\frac{d_{k}}{2}, \lambda\right\} & \text { if } \sum_{k \in M} \frac{d_{k}}{2} \geq E \\ d_{k}-\min \left\{\frac{d_{k}}{2}, \lambda\right\} & \text { if } \sum_{k \in M} \frac{d_{k}}{2} \leq E\end{cases}
$$

where $\lambda$ is chosen so that $\sum_{k \in M} \operatorname{Tb}_{k}(M, E, d)=E$. Second, it is well known that $\mathrm{Tb}$ satisfies CO. Thus, Theorem 5.1 remains valid if $\mathrm{BC}$ is replaced by CO.

\subsection{Markets}

Next, we introduce and discuss a number of properties that rules in $\mathcal{B B M}$ might satisfy. For the purpose of illustrating the properties, we henceforth consider the 2-regular market obtained from the market described in Example 2.1 by means of Eq. (2).

Example 5.1. Let $(\tilde{\mathbf{c}}, \tilde{w})$ be the 6-person three-sided market given by

$$
\left(\tilde{c}_{1}^{1}, \tilde{c}_{2}^{1} ; \tilde{c}_{1}^{2}, \tilde{c}_{2}^{2} ; \tilde{w}_{1}, \tilde{w}_{2}\right)=(50,55 ; 60,67 ; 130,120)
$$

\subsubsection{Core selection}

We start with a property that requires a rule to select payoffs that belong to the core of the assignment game.

CS A rule in $\mathcal{B B M}, \mathrm{f}$, satisfies core selection if for every $(\mathbf{c}, w) \in \mathcal{B B M}$,

$$
\mathrm{f}(\mathbf{c}, w) \in C\left(N, \omega^{\mathbf{c}, w}\right) .
$$


The above property requires a rule to share completely the spoils generated in the market, in such a way that no coalition of agents has incentives to search for an agreement on its own. Therefore, it can be considered a stability property. For the class of market games considered in this paper, CS can be spelled out in two different conditions. This is shown in the next lemma.

Lemma 5.1. A rule in $\mathcal{B B M}, \mathrm{f}$, satisfies $\mathrm{CS}$ if and only if for every $(\mathbf{c}, w) \in \mathcal{B B} \mathcal{M}$,

(i) $\mathbf{f}(\mathbf{c}, w)=t_{\mathbf{c}, w}+\mathcal{R}_{\mathbf{c}, w}\left(\left(f_{1}^{k}(\widetilde{\mathbf{c}}, \widetilde{w})\right)_{k \in M}\right)$,

(ii) $\mathbf{f}(\widetilde{\mathbf{c}}, \widetilde{w}) \in C\left(N, \omega^{\tilde{\mathbf{c}}, \tilde{w}}\right)$,

where $(\widetilde{\mathbf{c}}, \widetilde{w})$ is the 2-regular market associated with $(\mathbf{c}, w)$ according to Eq. (2).

Property CS has a further appeal when interpreted in terms of the money exchanged for goods by buyers and sellers. Indeed, consider a payoff vector $x=\left(\left(x_{i}^{k}\right)_{i \in N^{k}}\right)_{k \in M}$. To obtain a utility of $x_{i}^{k}$ when bargaining with a buyer, seller $i \in N^{k}$ must charge a price to the buyer equal to

$$
p_{i}^{k}(x)=x_{i}^{k}+c_{i}^{k}
$$

It turns out that there is a one-to-one correspondence between competitive prices and prices obtained from core allocations by means of Eq. (8) - see Remark 5.1 below. To define competitive prices, we need to introduce some concepts. Let $(\mathbf{c}, w) \in \mathcal{B B M}$ be a market and let $p=\left(\left(p_{i}^{k}\right)_{i \in N^{k}}\right)_{k \in M \backslash\{m\}}$ denote a vector of prices, one for each seller.

First, given a buyer $i_{m} \in N^{m}$, the demand set of $i_{m}$ at prices $p$, which we denote by $D_{i_{m}}(p)$, is the set of tuples $\left(i_{1}, \ldots, i_{m-1}\right) \in N^{1} \times \cdots \times N^{m-1}$ which maximize $w_{i_{m}}-\sum_{k \in M \backslash\{m\}} p_{i_{k}}^{k}$, whenever this last expression is positive. Otherwise, $D_{i_{m}}(p)$ is the empty set.

Second, a vector of prices $p$ is quasi-competitive if

(i) $p_{i}^{k} \geq c_{i}^{k}$, for every $i \in N^{k}$ and $k \in M \backslash\{m\}$, and

(ii) there is a matching $\mu \in \mathcal{M}\left(N^{1}, \ldots, N^{m}\right)$, which is said to be compatible with $p$, such that for every buyer $i_{m} \in N^{m}$,

$$
D_{i_{m}}(p) \neq \emptyset \Rightarrow \exists\left(i_{1}, \ldots, i_{m-1}\right) \in D_{i_{m}}(p) \text { such that }\left(i_{1}, \ldots, i_{m-1}, i_{m}\right) \in \mu \text {. }
$$

Third, a pair $(p, \mu) \in \prod_{k \in M \backslash\{m\}} \mathbb{R}^{N^{k}} \times \mathcal{M}\left(N^{1}, \ldots, N^{m}\right)$ is a competitive equilibrium if

(i) $p$ is a quasi-competitive vector of prices,

(ii) $\mu$ is a matching compatible with $p$, and

(iii) $p_{i}^{k}=c_{i}^{k}$ for every seller $i \in N^{k}$, with $k \in M \backslash\{m\}$, such that $i$ is assigned under $\mu$ to a buyer with an empty demand set.

Given a competitive equilibrium $(p, \mu)$, we say that the price $p$ is competitive. Building on part $(i)$ of Theorem 2.1, we can formulate the main result in Tejada (2010) for the class of markets considered in the paper, which reads as follows: 
Remark 5.1. Given $(\mathbf{c}, w) \in \mathcal{B B M}$, the following two statements are equivalent:

(a) $p$ is a vector of competitive prices,

(b) there exists $x \in C\left(N, \omega^{\mathbf{c}, w}\right)$ and $p(x)=\left(p^{1}(x), \ldots, p^{m-1}(x)\right) \in \mathbb{R}_{+}^{M \backslash\{m\}}$ such that for every $k \in M \backslash\{m\}$ and $i \in N^{k}$,

$$
p_{i}^{k}= \begin{cases}p^{k}(x)=x_{i}^{k}+c_{i}^{k} & \text { if } i \leq r, \\ c_{i}^{k} & \text { if } i>r .\end{cases}
$$

Whenever $x \in C\left(N, \omega^{\mathbf{c}, w}\right)$, it holds that $x_{i}^{k}+c_{i}^{k}=x_{j}^{k}+c_{j}^{k}$ for all $i, j \in N^{k}$ such that $i, j \leq r$. In what follows, for every $k \in M \backslash\{m\}$ we let $p^{k}(x) \in \mathbb{R}_{+}$denote the price of the commodity $k$ at allocation $x$. We stress that whenever $x$ is a core allocation of the assignment game, the vector $p(x)$, with components $p^{k}(x)=x_{1}^{k}+c_{1}^{k}$, collects the prices paid in any actual transaction that occurs in the market. Using Theorem 2.1, we find that the set of competitive prices of the market of Example 5.1 is

$$
\left\{\left(p^{1}, p^{2}\right): 53 \leq p^{1} \leq 55,65 \leq p^{2} \leq 67,120 \leq p^{1}+p^{2} \leq 122\right\}
$$

The remaining properties introduced in this section refer only to 2-regular markets.

\subsubsection{Vertical syndication-proof}

Suppose that prices in a market are determined according to a certain rule in $\mathcal{B B M}, \mathrm{f}$, which satisfies Cs. ${ }^{15}$ Let also $p(x)=\left(p^{1}(x), p^{2}(x)\right)$ be the current price level in the market of Example 5.1, with $x=\mathrm{f}(\tilde{\mathbf{c}}, \tilde{w})$. Then, assume that the following occurs: (i) the active firm in sector 1 buys the good from the active firm in sector 2 at price $p^{2}(x)$, (ii) the inactive firm in sector 1 buys the good from the inactive firm in sector 2 at price $p^{2}(x)$, and (iii) both firms in sector 1 offer the buyers a bundle composed of the two goods that each of them owns. The firms that sold their products no longer participate directly in the market. Other potential side-payments may nonetheless occur between all firms. This yields the following two-sided market:

Example 5.1 (cont.). Let $\left(N^{1}, N^{3}, \alpha^{1}, \tilde{w}\right)$ be the 2-sided 2-regular market given by

$$
\left(\alpha_{1}^{1}, \alpha_{2}^{1} ; \tilde{w}_{1}, \tilde{w}_{2}\right)=\left(50+p^{2}(x), 55+p^{2}(x) ; 130,120\right),
$$

where $x=\mathrm{f}(\tilde{\mathbf{c}}, \tilde{w})$.

The reduced market $\left(\alpha^{1}, \tilde{w}\right)$, with $\alpha^{1}=\left(\alpha_{1}^{1}, \alpha_{2}^{1}\right)$, captures the situation where one syndicate is formed between the two active firms and another between the two inactive firms. As the latter syndicate will not sell its bundle when prices are competitive, only one operative syndicate will actually form. In this new setting, a consumer can only buy the two-commodity bundled good but none of the goods separately. A cardinal question regarding the reduced market is: Who benefits and who loses in $\left(\alpha^{1}, \tilde{w}\right)$ with respect to the original market $(\tilde{\mathbf{c}}, \tilde{w})$ if prices are chosen according to $f$ in both markets? To answer this question, we have to compare sellers' and buyers' payoffs in

\footnotetext{
${ }^{15}$ We impose that $f$ satisfies CS to facilitate the explanation of the property.
} 
both settings. Doing so in real-world applications may be relevant for consumers, firms, and antitrust authorities.

The property below requires that buyers be indifferent to the two above situations.

2-VSP A rule in $\mathcal{B B} \mathcal{M}, \mathrm{f}$, satisfies vertical syndication-proofness for 2-regular markets if for every $\left(N^{1}, \ldots, N^{m}, \mathbf{c}, w\right) \in 2-\mathcal{B B} \mathcal{M}$ and $k \in M \backslash\{m\}$,

$$
\mathrm{f}^{m}\left(N^{1}, \ldots, N^{m}, \mathbf{c}, w\right)=\mathrm{f}^{m}\left(N^{k}, N^{m}, \alpha^{k}(\mathbf{c}, w, \mathrm{f}), w\right)
$$

where the reduced two-sided market $\left(N^{k}, N^{m}, \alpha^{k}(\mathbf{c}, w, f), w\right)$ is composed of the original sector of buyers, with valuations given by $w$, and a unique sector of sellers with $\operatorname{costs,} \alpha^{k}(\mathbf{c}, w, f)$, defined for every $i \in N^{k}$ by ${ }^{16}$

$$
\alpha_{i}^{k}(\mathbf{c}, w, f)=c_{i}^{k}+\sum_{l \in M \backslash\{k, m\}} p^{l}(\mathbf{f}(\mathbf{c}, w)) .
$$

Four comments are in order. First, the bargaining power in the 2-sided market $\left(\alpha^{k}(\mathbf{c}, w, \mathbf{f}), w\right)$ of the unique sector of firms coincides with the bargaining power of sector $k$ in the original market $(\mathbf{c}, w)$. Moreover, such a coincidence is independent of $\mathrm{f}$ by construction. Trivially, the same holds for the buyers. Second, 2-VSP relates the payoffs in a multi-sided market to the payoffs in a two-sided market. While the literature on bilateral assignment markets is large, comparatively few papers study multi-sided assignment markets. We are not aware of any property described in the literature which would connect the multilateral to the bilateral case. Third, if $\mathrm{f}$ satisfies CS, the set of 2-regular markets is closed under the reduced market operator.

Lemma 5.2. Let $(\mathbf{c}, w) \in 2-\mathcal{B B} \mathcal{M}$. If a rule, $\mathrm{f}$, satisfies $\mathrm{CS}$ then

(i) $\left(N^{k}, N^{m}, \alpha^{k}(\mathbf{c}, w, \mathrm{f}), w\right) \in 2-\mathcal{B B} \mathcal{M}$ for every $k \in M \backslash\{m\}$.

(ii) If $\left(N, \omega^{\mathbf{c}, w}\right)=\left(N, v_{0}\right)$ then $\left(N^{k} \cup N^{m}, \omega^{\alpha^{k}(\mathbf{c}, w, \mathbf{f}), w}\right)=\left(N, v_{0}\right)$.

Fourth and last, 2-VSP requires that the buyers' surplus is not affected by the creation of vertical syndicates. It is interesting also to investigate the incentives of sellers to create such syndicates. Lemma 5.3 below shows that when applied to a rule $\mathrm{f}$ that satisfies CS, 2-VSP requires that any active (resp. inactive) seller be indifferent to the following two options: (i) syndicating with $m-2$ other active (resp. inactive) sellers, each owning a different commodity, compensating them with the price prescribed by $\mathrm{f}$, and then bargaining directly with the buyers, and (ii) not creating such a syndicate.

Lemma 5.3. If a rule in $\mathcal{B B} \mathcal{M}, \mathrm{f}$, satisfies $\mathrm{CS}$ and 2 -VsP, then for every $(\mathbf{c}, w) \in 2-\mathcal{B B} \mathcal{M}$ and every $k \in M \backslash\{m\}$,

$$
\mathrm{f}^{k}(\mathbf{c}, w)=\mathrm{f}^{k}\left(\alpha^{k}(\mathbf{c}, w, \mathrm{f}), w\right) .
$$

\footnotetext{
${ }^{16}$ For the sake of understanding the property, we do not rename this unique sector of sellers, as it should be denoted by $N^{1}$ instead of $N^{k}$. An analogous comment applies to the sector of buyers. Also note that both the active seller and the inactive seller pay the same price. In particular, the inactive seller may be paying prices to other sellers that are below their costs.
} 
We have assumed that the sellers who are not present in the reduced market receive, according to 2-VSP, the payoff prescribed by $f$ in the original market - by means of the corresponding competitive price. Lemma 5.3 thus asserts that the total payoff of all active (or inactive) sellers should not be affected by the creation of the vertical syndicates. Indeed, it follows from Lemma 5.3 that under CS and 2-VSP, for every $(\mathbf{c}, w) \in 2-\mathcal{B B} \mathcal{M}$ and $i \in N^{k}$,

$$
\sum_{l \in M \backslash\{m\}} \mathrm{f}_{i}^{l}(\mathbf{c}, w)=\mathrm{f}_{i}^{k}\left(\alpha^{k}(\mathbf{c}, w, f), w\right)+\sum_{l \in M \backslash\{k, m\}} \mathrm{f}_{i}^{l}(\mathbf{c}, w) .
$$

As a consequence, under CS and 2-VSP there are no incentives for the creation of vertical syndicates in 2-regular markets. ${ }^{17}$

\subsubsection{Compromise in the bilateral case}

For bilateral markets, the core is a segment determined by both the buyers- and the sellers-optimal allocations. There seems to be a widespread consensus that the fairest solution to such a market game is the midpoint of the core. This allocation not only is the average between the two aforementioned polar allocations, but it also coincides with the nucleolus and the $\tau$-value - see Núnez and Rafels (2005). The following property requires that for every bilateral 2-regular market the midpoint of the core be selected.

2-CBS A rule in $\mathcal{B B M}, \mathrm{f}$, coincides with the canonical bilateral solution for bilateral 2-regular markets if for every 2 -sided market $(\mathbf{c}, w) \in 2-\mathcal{B B} \mathcal{M}$,

$$
\begin{aligned}
& f^{1}(\mathbf{c}, w)=\left(\frac{\left(w_{1}-c_{1}^{1}\right)_{+}+\left(w_{2}-c_{1}^{1}\right)_{+}-\left(w_{1}-c_{2}^{1}\right)_{+}}{2}, 0\right), \text { and } \\
& f^{2}(\mathbf{c}, w)=\left(\frac{\left(w_{1}-c_{1}^{1}\right)_{+}+\left(w_{1}-c_{2}^{1}\right)_{+}-\left(w_{2}-c_{1}^{1}\right)_{+}}{2}, 0\right)
\end{aligned}
$$

\subsubsection{Anonymity with respect to the bargaining power}

In Section 2 we have defined the bargaining power of each sector in a 2-regular market. The property below requires that in 2-regular markets, traders - be they firms or consumers - be treated equally with regard to bargaining power.

2-ABP A rule in $\mathcal{B B M}, \mathrm{f}$, satisfies anonymity with respect to bargaining power for 2-regular markets if for every two markets $(\mathbf{c}, w),(\hat{\mathbf{c}}, \hat{w}) \in \mathcal{2}-\mathcal{B B} \mathcal{M}$ with the same total surplus $w_{1}-\sum_{k \in M \backslash\{m\}} c_{1}^{k}=$ $\hat{w}_{1}-\sum_{k \in M \backslash\{m\}} \hat{c}_{1}^{k}$, if there is $k \in M \backslash\{m\}$ such that $b^{k}(\mathbf{c}, w)=b^{m}(\hat{\mathbf{c}}, \hat{w}), b^{m}(\mathbf{c}, w)=b^{k}(\hat{\mathbf{c}}, \hat{w})$, and $b^{l}(\mathbf{c}, w)=b^{l}(\hat{\mathbf{c}}, \hat{w})$ for all $l \in M \backslash\{k, m\}$, then

$$
f^{l}(\hat{\mathbf{c}}, \hat{w})= \begin{cases}\mathrm{f}^{m}(\mathbf{c}, w) & \text { if } l=k, \\ \mathrm{f}^{k}(\mathbf{c}, w) & \text { if } l=m, \\ \mathrm{f}^{l}(\mathbf{c}, w) & \text { otherwise }\end{cases}
$$

\footnotetext{
${ }^{17}$ At this point, the question whether or not to require Eq. (10) to hold with a weak inequality arises. The answer to this question falls outside the scope of the current paper. In Knudsen and Østerdal (2012) merging and splitting incentives are studied separately for arbitrary cooperative games.
} 


\section{Exploiting the link: a characterization result}

In this section, we do two things. First, we study the relation between the properties presented in the previous section for bankruptcy problems and markets. Second, we characterize the unique rule that satisfies, among other properties, our proposed notion of vertical syndication-proofness.

\subsection{Translating properties}

First, we study the translation of properties from the domain of markets to the domain of bankruptcy problems.

Proposition 6.1. Let $\mathrm{f}$ be a rule in $\mathcal{B B M}$ and $\Psi$ the mapping defined in Eq. (7).

(i) If $\mathrm{f}$ satisfies $\mathrm{CS}$, then $\Psi(\mathrm{f})$ satisfies $\mathrm{WD}$.

(ii) If $\mathrm{f}$ satisfies 2 -CBS, then $\Psi(\mathrm{f})$ satisfies $\mathrm{CG}$.

(iii) If $\mathrm{f}$ satisfies $\mathrm{CS}, 2-\mathrm{CBS}, 2-\mathrm{VSP}$, and 2-ABP, then $\Psi(\mathrm{f})$ satisfies $\mathrm{BC}$.

Proof.

Let $\mathrm{fb}=\Psi(\mathrm{f})$ and $(E, d) \in \mathcal{B P}$. Throughout the proof, let also $N^{1}, \ldots, N^{m}$ denote the sets of agents of the market obtained from $(E, d)$ via Eq. (4) and $N=N^{1} \cup \cdots \cup N^{m}$. Recall that $(\mathbf{c}(E, d), w(E, d)) \in 2-\mathcal{B B M}$.

For part (i), assume that $\mathrm{f}$ satisfies CS. Then,

$$
\sum_{k \in M} \mathrm{fb}_{k}(E, d)=\sum_{k \in M} \mathrm{f}_{1}^{k}(\mathbf{c}(E, d), w(E, d))=\omega^{\mathbf{c}(E, d), w(E, d)}(N)=E,
$$

where the first equality holds by definition of $\Psi$ in Eq. (7), the second equality holds since $\mathrm{f}$ satisfies CS, and the last equality holds by Eq. (4). Moreover, since a player's payoff in the core is bounded from below by her stand-alone worth and from above by her marginal contribution to the grand coalition, we obtain that for every $k \in M \backslash\{m\}$,

$$
\begin{aligned}
0 & \leq \mathrm{fb}_{k}(E, d)=\mathrm{f}_{1}^{k}(\mathbf{c}(E, d), w(E, d)) \leq \omega^{\mathbf{c}(E, d), w(E, d)}(N)-\omega^{\mathbf{c}(E, d), w(E, d)}\left(N \backslash\left\{1^{k}\right\}\right) \\
& =\left(w_{1}(E, d)-\sum_{l \in M \backslash\{m\}} c_{1}^{l}(E, d)\right)_{+}-\left(w_{1}(E, d)-\sum_{l \in M \backslash\{k, m\}} c_{1}^{l}(E, d)-c_{2}^{k}(E, d)\right)_{+} \\
& \leq c_{2}^{k}(E, d)-c_{1}^{k}(E, d)=d_{k},
\end{aligned}
$$

and

$$
\begin{aligned}
0 & \leq \mathrm{fb}_{m}(E, d)=\mathrm{f}_{1}^{m}(\mathbf{c}(E, d), w(E, d)) \leq \omega^{\mathbf{c}(E, d), w(E, d)}(N)-\omega^{\mathbf{c}(E, d), w(E, d)}\left(N \backslash\left\{1^{m}\right\}\right) \\
& =\left(w_{1}(E, d)-\sum_{l \in M \backslash\{m\}} c_{1}^{l}(E, d)\right)_{+}-\left(w_{2}(E, d)-\sum_{l \in M \backslash\{m\}} c_{1}^{l}(E, d)\right)_{+} \\
& \leq w_{1}(E, d)-w_{2}(E, d)=d_{m},
\end{aligned}
$$

where $1^{k}$ denotes the first agent in sector $k \in M$. Hence, $\Psi(\mathrm{f})$ satisfies WD. 
For part (ii), assume that $\mathrm{f}$ satisfies 2-CBS. Then, let $(M, E, d) \in \mathcal{B P}$ be such that $M=\{1,2\}$. By Eq. (4) and the fact that $f$ satisfies 2-CBS, it follows that

$$
\begin{aligned}
\mathrm{fb}_{1}(E, d) & =\frac{1}{2}\left(\left(w_{1}(E, d)-c_{1}^{1}(E, d)\right)_{+}+\left(w_{2}(E, d)-c_{1}^{1}(E, d)\right)_{+}-\left(w_{1}(E, d)-c_{2}^{1}(E, d)\right)_{+}\right) \\
& =\frac{1}{2}\left(E+\left(E-d_{2}\right)_{+}-\left(E-d_{1}\right)_{+}\right) .
\end{aligned}
$$

We can repeat the same argument for the second player in the bankruptcy problem and obtain $\mathrm{fb}_{2}(E, d)=\frac{1}{2}\left(E+\left(E-d_{1}\right)_{+}-\left(E-d_{2}\right)_{+}\right)$. Hence, $\Psi(\mathrm{f})$ satisfies CG.

Finally, for part (iii) assume that $f$ satisfies CS, 2-CBS, 2-VSP, and 2-ABP. In the following, we show that $\mathrm{fb}$ is bilaterally consistent. To ease the notation, let henceforth $y_{k}=\mathrm{fb}_{k}(M, E, d)$ for every $k \in M$. Let $S \subseteq M$ be such that $|S|=2$. We distinguish two cases.

Case A: $m \in S$

Accordingly, $S=\{k, m\}$ for some $k \in M \backslash\{m\}$. For $l \in\{k, m\}$, let $z_{l}=\mathrm{fb}_{l}\left(\{k, m\}, y_{k}+\right.$ $\left.y_{m},\left(d_{k}, d_{m}\right)\right)$. On the one hand, by Eq. (4),

$$
\begin{aligned}
\mathbf{c}\left(y_{k}+y_{m},\left(d_{k}, d_{m}\right)\right) & =\left(d_{m}, d_{m}+d_{k}\right) \quad \text { and } \\
w\left(y_{k}+y_{m},\left(d_{k}, d_{m}\right)\right) & =\left(y_{k}+y_{m}+d_{m}, y_{k}+y_{m}\right) .
\end{aligned}
$$

By definition of $\Psi$ and the fact that $f$ satisfies 2-CBS, we obtain

$$
\begin{aligned}
z_{k} & =\frac{1}{2}\left(y_{k}+y_{m}+\left(y_{k}+y_{m}-d_{m}\right)_{+}-\left(y_{k}+y_{m}-d_{k}\right)_{+}\right) \quad \text { and } \\
z_{m} & =\frac{1}{2}\left(y_{k}+y_{m}+\left(y_{k}+y_{m}-d_{k}\right)_{+}-\left(y_{k}+y_{m}-d_{m}\right)_{+}\right) .
\end{aligned}
$$

On the other hand, by definition of $\Psi$ in Eq. (7), the fact that $f$ satisfies 2-VSP, and Lemma 5.3, we have for $l \in\{k, m\}$,

$$
y_{l}=\mathrm{f}_{1}^{l}(\mathbf{c}(E, d), w(E, d))=\mathrm{f}_{1}^{l}\left(\alpha^{k}(\mathbf{c}(E, d), w(E, d), \mathbf{f}), w(E, d)\right) .
$$

Note that the right-hand side of Eq. (12) involves a bilateral market. By Eq. (9),

$$
\alpha^{k}(\mathbf{c}(E, d), w(E, d), \mathbf{f})=\left(\begin{array}{l}
c_{1}^{k}(E, d)+\sum_{l \in M \backslash\{k, m\}}\left[c_{1}^{l}(E, d)+\mathrm{f}_{1}^{l}(\mathbf{c}(E, d), w(E, d))\right] \\
c_{2}^{k}(E, d)+\sum_{l \in M \backslash\{k, m\}}\left[c_{1}^{l}(E, d)+\mathrm{f}_{1}^{l}(\mathbf{c}(E, d), w(E, d))\right] .
\end{array}\right)
$$

Since $f$ satisfies CS,

$$
\sum_{l \in M \backslash\{k, m\}} \mathrm{f}_{1}^{l}(\mathbf{c}(E, d), w(E, d))=\omega^{\mathbf{c}(E, d), w(E, d)}(N)-\sum_{l \in\{k, m\}} \mathrm{f}_{1}^{l}(\mathbf{c}(E, d), w(E, d))=E-y_{k}-y_{m},
$$

where the last equality holds by the definition of the assignment game and Eq. (4). Then, using Eq. (4) and the equation above, Eq. (13) becomes

$$
\alpha^{k}(\mathbf{c}(E, d), w(E, d), \mathbf{f})=\left(\begin{array}{c}
d_{m}+E-y_{k}-y_{m} \\
d_{m}+d_{k}+E-y_{k}-y_{m}
\end{array}\right) .
$$

Finally, since f satisfies 2-CBS, Eqs. (12) and (14) yield

$$
\begin{aligned}
y_{k} & =\frac{1}{2}\left(y_{k}+y_{m}+\left(y_{k}+y_{m}-d_{m}\right)_{+}-\left(y_{k}+y_{m}-d_{k}\right)_{+}\right) \quad \text { and } \\
y_{m} & =\frac{1}{2}\left(y_{k}+y_{m}+\left(y_{k}+y_{m}-d_{k}\right)_{+}-\left(y_{k}+y_{m}-d_{m}\right)_{+}\right) .
\end{aligned}
$$


That is, we have proved that $y_{l}=z_{l}$ for $l \in\{k, m\}$.

Case B: $m \notin S$

Hence, $S=\{k, l\}$ with $k, l \in M \backslash\{m\}$ and $k \neq l$. Consider the auxiliary bankruptcy problem $\left(E, d^{\prime}\right) \in \mathcal{B P}$ defined by $d_{l}^{\prime}=d_{m}, d_{m}^{\prime}=d_{l}$, and $d_{h}^{\prime}=d_{h}$ for all $h \in M \backslash\{l, m\}$. Denote $y_{h}^{\prime}=\mathrm{fb}_{h}\left(E, d^{\prime}\right)$ for all $h \in M$. Let $\mathbf{c}^{\prime}=\mathbf{c}\left(E, d^{\prime}\right)$ and $w^{\prime}=w\left(E, d^{\prime}\right)$. Since $\mathrm{f}$ satisfies 2-ABP, we can apply such a property to markets $(\mathbf{c}(E, d), w(E, d))$ and $\left(\mathbf{c}^{\prime}, w^{\prime}\right)$ to obtain

$$
\begin{aligned}
& y_{k}=\mathrm{f}_{1}^{k}(\mathbf{c}(E, d), w(E, d))=\mathrm{f}_{1}^{k}\left(\mathbf{c}^{\prime}, w^{\prime}\right)=y_{k}^{\prime} \quad \text { and } \\
& y_{l}=\mathrm{f}_{l}^{l}(\mathbf{c}(E, d), w(E, d))=\mathrm{f}_{1}^{m}\left(\mathbf{c}^{\prime}, w^{\prime}\right)=y_{m}^{\prime} .
\end{aligned}
$$

In particular, note that

$$
y_{k}+y_{l}=y_{k}^{\prime}+y_{m}^{\prime} .
$$

Moreover, let $z_{h}^{\prime}=\mathrm{fb}_{h}\left(\{k, m\}, y_{k}^{\prime}+y_{m}^{\prime},\left(d_{k}^{\prime}, d_{m}^{\prime}\right)\right)$ for $h \in\{k, m\}$. Then, from Case A we know that

$$
y_{k}^{\prime}=z_{k}^{\prime} \quad \text { and } \quad y_{m}^{\prime}=z_{m}^{\prime}
$$

Since by part (ii) fb satisfies CG, we have

$$
\begin{aligned}
z_{k}^{\prime} & =\frac{1}{2}\left(y_{k}^{\prime}+y_{m}^{\prime}+\left(y_{k}^{\prime}+y_{m}^{\prime}-d_{m}^{\prime}\right)_{+}-\left(y_{k}^{\prime}+y_{m}^{\prime}-d_{k}^{\prime}\right)_{+}\right) \quad \text { and } \\
z_{m}^{\prime} & =\frac{1}{2}\left(y_{k}^{\prime}+y_{m}^{\prime}+\left(y_{k}^{\prime}+y_{m}^{\prime}-d_{k}^{\prime}\right)_{+}-\left(y_{k}^{\prime}+y_{m}^{\prime}-d_{m}^{\prime}\right)_{+}\right) .
\end{aligned}
$$

Next, let $z_{h}=\mathrm{fb}_{h}\left(\{k, l\}, y_{k}+y_{l},\left(d_{k}, d_{l}\right)\right)$ for $h \in\{k, l\}$. Again because fb satisfies CG,

$$
\begin{aligned}
z_{k} & =\frac{1}{2}\left(y_{k}+y_{l}+\left(y_{k}+y_{l}-d_{l}\right)_{+}-\left(y_{k}+y_{l}-d_{k}\right)_{+}\right) \quad \text { and } \\
z_{l} & =\frac{1}{2}\left(y_{k}+y_{l}+\left(y_{k}+y_{l}-d_{k}\right)_{+}-\left(y_{k}+y_{l}-d_{l}\right)_{+}\right) .
\end{aligned}
$$

Then, by Eq. (16) and taking into account that $d_{l}^{\prime}=d_{m}$ and $d_{m}^{\prime}=d_{l}$, it follows that

$$
z_{k}^{\prime}=z_{k} \quad \text { and } z_{m}^{\prime}=z_{l}
$$

Lastly, it follows from Eqs. (15), (17), and (18) that $y_{h}=z_{h}$ for $l \in\{k, l\}$, so the proof is complete.

Second, we study the translation of properties from the domain of bankruptcy problems to that of markets.

Proposition 6.2. Let $\mathrm{fb}$ be a rule in $\mathcal{B P}$ and $\Phi$ the mapping defined in Eq. (6).

(i) If $\mathrm{fb}$ satisfies $\mathrm{CG}$, then $\Phi(\mathrm{fb})$ satisfies 2-CBS.

(ii) If $\mathrm{fb}$ satisfies $\mathrm{WD}, \mathrm{CG}$, and $\mathrm{BC}$, then $\Phi(\mathrm{fb})$ satisfies $\mathrm{CS}, 2-\mathrm{VSP}$, and 2-ABP.

\section{Proof.}

Let $\mathrm{fb}$ be a rule in $\mathcal{B P}$ and $\mathrm{f}=\Phi(\mathrm{fb})$. By Lemma 5.1 and construction of $\Phi$ in Eq. (6), it follows that $f$ satisfies CS if it associates a core allocation with every 2-regular market. Since 2-CBS, 2-vSP, and 2-ABP only apply to 2-regular markets, we henceforth assume that $(\mathbf{c}, w) \in 2-\mathcal{B B M}$. Note in particular that by construction of $\Phi$ in Eq. (6), it follows that for every sector $k \in M, \mathrm{f}_{2}^{k}(\mathbf{c}, w)=0$. 
For part $(i)$, let $\left(N^{1}, N^{2}, \mathbf{c}, w\right) \in 2-\mathcal{B B} \mathcal{M}$ be a bilateral 2-regular market. Since fb satisfies $\mathrm{CG}$,

$$
\begin{aligned}
\mathbf{f}_{1}^{1}(\mathbf{c}, w)=\mathrm{fb}_{1}(E(\mathbf{c}, w), d(\mathbf{c}, w)) & =\frac{1}{2}\left(E(\mathbf{c}, w)+\left(E(\mathbf{c}, w)-d_{2}(\mathbf{c}, w)\right)_{+}-\left(E(\mathbf{c}, w)-d_{1}(\mathbf{c}, w)\right)_{+}\right) \\
& =\frac{1}{2}\left(\left(w_{1}-c_{1}^{1}\right)_{+}+\left(w_{2}-c_{1}^{1}\right)_{+}-\left(w_{1}-c_{2}^{1}\right)_{+}\right) .
\end{aligned}
$$

Following an analogous argument for the sector of buyers yields

$$
\mathbf{f}_{1}^{2}(\mathbf{c}, w)=\frac{1}{2}\left(\left(w_{1}-c_{1}^{1}\right)_{+}+\left(w_{1}-c_{2}^{1}\right)_{+}-\left(w_{2}-c_{1}^{1}\right)_{+}\right) .
$$

Hence, $f$ satisfies 2-CBS.

For part (ii), suppose that fb satisfies WD, CG, and BC. By Theorem 5.1, fb selects the nucleolus of the bankruptcy game induced by any bankruptcy problem. If $r(\mathbf{c}, w)=0$, we have $\left(N, \omega^{\mathbf{c}, w}\right)=$ $\left(N, v_{0}\right)$, so f satisfies CS, 2-VSP, and 2-ABP. We thus focus for the rest of the proof on the case where $r(\mathbf{c}, w) \neq 0$. First, we show that $\mathrm{f}$ satisfies CS. Note that part $(i)$ of Theorem 2.1 implies that

$$
\mathrm{f}(\mathbf{c}, w) \in C\left(N, \omega^{\mathbf{c}, w}\right) \Leftrightarrow\left(\mathrm{f}_{1}^{k}(\mathbf{c}, w)\right)_{k \in M} \in C\left(M, v^{\mathbf{c}, w}\right) \Leftrightarrow \mathrm{fb}(E(\mathbf{c}, w), d(\mathbf{c}, w)) \in C\left(M, v^{\mathbf{c}, w}\right) .
$$

Therefore, from Theorem I - and its proof - and the fact that the nucleolus is always a core allocation, we deduce that $\mathbf{f}(\mathbf{c}, w) \in C\left(N, \omega^{\mathbf{c}, w}\right)$.

Second, to show that $\mathrm{f}$ satisfies 2-VSP, let $y_{l}=\mathrm{f}_{1}^{l}(\mathbf{c}, w)$ for all $l \in M$. By construction of $\Phi$ in Eq. (6), we have $y=\mathrm{fb}(E(\mathbf{c}, w), d(\mathbf{c}, w))$. Let $\left(N^{k}, N^{m}, \alpha^{k}(\mathbf{c}, w, f), w\right)$ be the reduced bilateral 2-regular market obtained from $(\mathbf{c}, w)$, with $k \in M \backslash\{m\}$. Let $z_{l}=\mathrm{f}_{1}^{l}\left(N^{k}, N^{m}, \alpha^{k}(\mathbf{c}, w, f), w\right)$ for $l \in\{k, m\}$. By definition of the costs of the reduced market - see Eq. (9)-,

$$
\alpha_{1}^{k}(\mathbf{c}, w, \mathbf{f})=c_{1}^{k}+\sum_{l \in M \backslash\{k, m\}}\left(c_{1}^{l}+y_{l}\right)=w_{1}-y_{k}-y_{m},
$$

where the last holds since $f$ satisfies CS. Similarly,

$$
\alpha_{2}^{k}(\mathbf{c}, w, \mathbf{f})=w_{1}+c_{2}^{k}-c_{1}^{k}-y_{k}-y_{m} .
$$

Using Eq. (5), we obtain

$$
\begin{aligned}
E\left(\alpha^{k}(\mathbf{c}, w, f), w\right) & =w_{1}-\alpha_{1}^{k}(\mathbf{c}, w, f)=y_{k}+y_{m}, \\
d_{k}\left(\alpha^{k}(\mathbf{c}, w, f), w\right) & =c_{2}^{k}-c_{1}^{k}=d_{k}(\mathbf{c}, w), \quad \text { and } \\
d_{m}\left(\alpha^{k}(\mathbf{c}, w, f), w\right) & =w_{1}-w_{2}=d_{m}(\mathbf{c}, w) .
\end{aligned}
$$

By construction of $\Phi$ in Eq. (6) and the fact that fb satisfies BC, it follows from the above equations that for $l \in\{k, m\}$,

$$
z_{l}=\mathrm{fb}_{l}\left(\{k, m\}, y_{k}+y_{m},\left(d_{k}(\mathbf{c}, w)\right), d_{m}(\mathbf{c}, w)\right)=\mathrm{fb}_{l}(M, E(\mathbf{c}, w), d(\mathbf{c}, w))=y_{l} .
$$

Thus, $f$ satisfies 2-VSP.

Third, to show that $\mathrm{f}$ satisfies 2-ABP, let $(\mathbf{c}, w)$ and $(\hat{\mathbf{c}}, \hat{w})$ be as in the definition of 2-ABP. Then $\left(N, \omega^{\mathbf{c}, w}\right)$ is obtained from $\left(N, \omega^{\hat{\mathbf{c}}, \hat{w}}\right)$ by exchanging the roles of players in the sectors $k$ and $m$. Since the nucleolus is an anonymous solution concept, part $(i i)$ of Theorem 2.1 implies that $f$ satisfies $2-\mathrm{ABP}^{18}$

\footnotetext{
${ }^{18}$ A point- or set-valued solution concept $f$ is anonymous if for all $(N, v) \in \mathcal{G}$ and $i \in N$, it holds that $f_{\pi(i)}(N, \pi v)=$ $f_{i}(N, v)$, where $\pi: N \rightarrow N$ is a permutation and $\pi v(S)=v(\pi(S))$ for all $S \subseteq N$.
} 


\subsection{The main result}

Next, we prove the paper's main result. It is convenient to define the rule obtained by translating the Talmud Bankruptcy Rule.

Definition 6.1. The Talmud Assignment Rule, $\mathrm{T}$, is the rule in $\mathcal{B B M}$ defined as the transformation using $\Phi$ of the Talmud Bankruptcy Rule, i.e., $\mathrm{T}=\Phi(\mathrm{Tb})$.

We are now in a position to state and prove the theorem.

Theorem II. The Talmud Assignment Rule is the unique rule in $\mathcal{B B M}$ that satisfies $\mathrm{Cs}, 2$-VSP, 2-ABP, and 2-CBS. Moreover, it selects for every market the nucleolus of the associated assignment game.

\section{Proof.}

The proof proceeds in three steps. First, we show that if there is a rule in $\mathcal{B B M}$ satisfying CS, 2-VSP, 2-CBS, and 2-ABP, then it must be unique. Second, we show that there is a rule in $\mathcal{B B} \mathcal{M}$, namely $T$, that satisfies the four properties. Third, we show that these four properties do in fact single out the nucleolus of the market games in $\mathcal{B B G}$.

\section{Step 1: Uniqueness}

Let $\mathrm{f}$ be a rule in $\mathcal{B B M}$ that satisfies CS, 2-VSP, 2-CBS, and 2-ABP. First, let $\mathcal{H}$ be the set of 2-regular markets obtained from bankruptcy problems via Eq. (4), i.e.,

$$
\mathcal{H}=\{(\mathbf{c}, w) \in 2-\mathcal{B B} \mathcal{M}: \exists(E, d) \in \mathcal{B P} \text { s.t. }(\mathbf{c}, w)=(\mathbf{c}(E, d), w(E, d))\}
$$

Since the mappings $\mathbf{c}(E, d)$ and $w(E, d)$ are not surjective, $\mathcal{H} \subsetneq 2-\mathcal{B B M}$. We show that $\mathrm{f}$ is the only rule in $\mathcal{H}$ satisfying the four properties. Indeed, let $(\mathbf{c}, w) \in \mathcal{H}$. Then, there is $(E, d) \in \mathcal{B P}$ such that for all $k \in M$,

$$
\mathrm{f}_{1}^{k}(\mathbf{c}, w)=\mathrm{f}_{1}^{k}(\mathbf{c}(E, d), w(E, d))=\Psi(\mathbf{f})_{k}(E, d),
$$

where the last equality holds by definition of $\Psi$ in Eq. (7). Since $f$ satisfies CS, 2-VsP, 2-CBS, and 2-ABP, it follows from Proposition 6.1 that $\Psi(\mathrm{f})$ satisfies WD, BC, and CG, which implies by Theorem 5.1 the uniqueness of the payoffs in Eq. (20).

Second, from Lemma 5.1, we know that a rule in $\mathcal{B B M}$ that satisfies CS is uniquely defined for all $(\mathbf{c}, w) \in \mathcal{B B M}$ if and only if it is uniquely defined for all $(\mathbf{c}, w) \in 2-\mathcal{B B M}$. Thus, henceforth, we restrict our attention to $2-\mathcal{B B M}$. Moreover, CS also implies that for all $(\mathbf{c}, w) \in 2-\mathcal{B B M}$ and $k \in M$, $\mathrm{f}_{2}^{k}(\mathbf{c}, w)=0$. As a consequence, we only need to show the uniqueness of $\mathrm{f}_{1}^{1}(\mathbf{c}, w), \ldots, \mathrm{f}_{1}^{m}(\mathbf{c}, w)$.

Third, we define several binary relations in $2-\mathcal{B B M}$ based on the relation between the markets in the definition of 2-ABP. Consider two arbitrary 2-regular markets $(\mathbf{c}, w),(\hat{\mathbf{c}}, \hat{w}) \in 2-\mathcal{B B M}$. On the one hand, let $k \in M \backslash\{m\}$. Then,

$$
(\mathbf{c}, w) \sim^{k}(\hat{\mathbf{c}}, \hat{w}) \Leftrightarrow\left\{\begin{array}{l}
w_{1}-\sum_{l \in M \backslash\{m\}} c_{1}^{l}=\hat{w}_{1}-\sum_{l \in M \backslash\{m\}} \hat{c}_{1}^{l}, \\
c_{2}^{k}-c_{1}^{k}=\hat{w}_{1}-\hat{w}_{2}, \\
w_{1}-w_{2}=\hat{c}_{2}^{k}-\hat{c}_{1}^{k}, \text { and } \\
c_{2}^{l}-c_{1}^{l}=\hat{c}_{2}^{l}-\hat{c}_{1}^{l} \text { for every } l \in M \backslash\{k, m\} .
\end{array}\right.
$$


Similarly,

$$
(\mathbf{c}, w) \sim^{m}(\hat{\mathbf{c}}, \hat{w}) \Leftrightarrow\left\{\begin{array}{l}
w_{1}-\sum_{k \in M \backslash\{m\}} c_{1}^{k}=\hat{w}_{1}-\sum_{k \in M \backslash\{m\}} \hat{c}_{1}^{k}, \\
w_{1}-w_{2}=\hat{w}_{1}-\hat{w}_{2}, \text { and } \\
c_{2}^{l}-c_{1}^{l}=\hat{c}_{2}^{l}-\hat{c}_{1}^{l} \text { for every } l \in M \backslash\{m\} .
\end{array}\right.
$$

On the other hand,

$$
(\mathbf{c}, w) \sim(\hat{\mathbf{c}}, \hat{w}) \Leftrightarrow(\mathbf{c}, w) \sim^{k}(\hat{\mathbf{c}}, \hat{w}) \text { for some } k \in M .
$$

Note that $\sim$ is an equivalence relation. For convenience, for a given $k \in M$, let $\pi^{k}$ be the permutation of $M$ defined by $\pi^{k}(k)=m, \pi^{k}(m)=k$, and for every $l \in M \backslash\{k, m\}, \pi^{k}(l)=l$.

Fourth, given an arbitrary $(\mathbf{c}, w) \in 2-\mathcal{B B} \mathcal{M}$, let $\left(\mathbf{c}^{\prime}, w^{\prime}\right) \in \mathcal{H}$ be defined by ${ }^{19}$

$$
\mathbf{c}^{\prime}=\mathbf{c}(E(\mathbf{c}, w), d(\mathbf{c}, w)) \text { and } w^{\prime}=w(E(\mathbf{c}, w), d(\mathbf{c}, w)) .
$$

It is straightforward to verify that the sectors games associated with the above markets coincide, i.e., that $\left(M, v^{\mathbf{c}, w}\right)=\left(M, v^{\mathbf{c}^{\prime}, w^{\prime}}\right)$.

The remainder of the proof is devoted to showing that for every $(\mathbf{c}, w) \in 2-\mathcal{B B M} \backslash \mathcal{H}, \mathrm{f}(\mathbf{c}, w)=$ $\mathrm{f}\left(\mathbf{c}^{\prime}, w^{\prime}\right)$. To that purpose, we assume on the contrary that there exists a market $\left(\mathbf{c}^{*}, w^{*}\right) \in 2-\mathcal{B B} \mathcal{M} \backslash \mathcal{H}$ such that

$$
\mathrm{f}\left(\mathbf{c}^{*}, w^{*}\right) \neq \mathrm{f}\left(\left(\mathbf{c}^{*}\right)^{\prime},\left(w^{*}\right)^{\prime}\right),
$$

where $\left(\left(\mathbf{c}^{*}\right)^{\prime},\left(w^{*}\right)^{\prime}\right) \in \mathcal{H}$ is obtained from $\left(\mathbf{c}^{*}, w^{*}\right)$ as in Eq. (21). We shall reach a contradiction.

Consider now the rule $\mathrm{g}$ in $2-\mathcal{B B M}$ defined for every $(\mathbf{c}, w) \in \mathcal{2}-\mathcal{B B M}$ and $l \in M$ as follows:

$$
\mathrm{g}^{l}(\mathbf{c}, w)= \begin{cases}\mathrm{f}^{\pi^{k}(l)}\left(\mathbf{c}^{*}, w^{*}\right) & \text { if }(\mathbf{c}, w) \sim^{k}\left(\mathbf{c}^{*}, w^{*}\right), \text { for some } k \in M, \\ \mathrm{f}^{l}\left(\mathbf{c}^{\prime}, w^{\prime}\right) & \text { if }(\mathbf{c}, w) \nsim\left(\mathbf{c}^{*}, w^{*}\right), \text { where }\left(\mathbf{c}^{\prime}, w^{\prime}\right) \in \mathcal{H} \text { is as defined in Eq. (21). }\end{cases}
$$

We shall show that g satisfies CS, 2-VSP, 2-ABP, and 2-CBS. The following two claims will be helpful.

Claim A. Let $(\mathbf{c}, w),(\overline{\mathbf{c}}, \bar{w}) \in 2-\mathcal{B B M}$ be two 2-sided 2-regular markets with the same total surplus and same bargaining powers, i.e., $w_{1}-c_{1}^{1}=\bar{w}_{1}-\bar{c}_{1}^{1}, b^{1}(\mathbf{c}, w)=b^{1}(\overline{\mathbf{c}}, \bar{w})$, and $b^{2}(\mathbf{c}, w)=b^{2}(\overline{\mathbf{c}}, \bar{w})$. If $\mathrm{f}$ is a rule in 2-BBM satisfying 2 -CBS, then $\mathrm{f}(\mathbf{c}, w)=\mathrm{f}(\overline{\mathbf{c}}, \bar{w})$.

Claim B. Let $(\mathbf{c}, w),(\overline{\mathbf{c}}, \bar{w}) \in 2-\mathcal{B B M}$ be two 2-sided 2-regular markets with the same total surplus and switched bargaining powers, i.e., $w_{1}-c_{1}^{1}=\bar{w}_{1}-\bar{c}_{1}^{1}, b^{1}(\mathbf{c}, w)=b^{2}(\overline{\mathbf{c}}, \bar{w})$, and $b^{2}(\mathbf{c}, w)=b^{1}(\overline{\mathbf{c}}, \bar{w})$. If $\mathrm{f}$ is a rule in 2-BBM satisfying 2 -CBS, then $\mathrm{f}^{1}(\mathbf{c}, w)=\mathrm{f}^{2}(\overline{\mathbf{c}}, \bar{w})$ and $\mathrm{f}^{2}(\mathbf{c}, w)=\mathrm{f}^{1}(\overline{\mathbf{c}}, \bar{w})$.

Note that because $\mathbf{f}$ satisfies 2 -CBS, the market $\left(\mathbf{c}^{*}, w^{*}\right)$ must have at least three sides, i.e., $m>2$. Otherwise Claim A applied to rule $\mathrm{f}$ and markets $\left(\mathbf{c}^{*}, w^{*}\right)$ and $\left(\left(\mathbf{c}^{*}\right)^{\prime},\left(w^{*}\right)^{\prime}\right)$ contradicts Eq. (22).

Next we proceed to prove that $\mathrm{g}$ satisfies the four properties. With regard to CS, note that whenever $(\mathbf{c}, w) \sim^{k}(\hat{\mathbf{c}}, \hat{w}),\left(M, v^{\hat{\mathbf{c}}, \hat{w}}\right)$ can be obtained from $\left(M, v^{\mathbf{c}, w}\right)$ by permuting the players according to $\pi^{k}$. Then, $\mathrm{g}$ satisfies CS by Theorem 2.1 and the following facts: $\mathrm{f}$ satisfies CS and the core is an anonymous solution concept. Moreover, $g$ satisfies 2-CBS trivially because $f$ does so. It is also

\footnotetext{
${ }^{19}$ We abuse notation and do not write the dependence of $\mathbf{c}^{\prime}$ and $w^{\prime}$ on the market $(\mathbf{c}, w)$. This spares us the use of some cumbersome expressions.
} 
straightforward to verify that by construction, $g$ satisfies 2-ABP. Then, it remains to prove that $g$ satisfies 2-VSP. Let $(\mathbf{c}, w) \in 2-\mathcal{B B M}$ and $k \in M \backslash\{m\}$. We distinguish three cases.

Case A: $(\mathbf{c}, w) \nsim\left(\mathbf{c}^{*}, w^{*}\right)$.

Then,

$$
\mathrm{g}^{m}(\mathbf{c}, w)=\mathrm{f}^{m}\left(\mathbf{c}^{\prime}, w^{\prime}\right)=\mathrm{f}^{m}\left(\alpha^{k}\left(\mathbf{c}^{\prime}, w^{\prime}, \mathrm{f}\right), w^{\prime}\right)=\mathrm{f}^{m}\left(\left(\alpha^{k}(\mathbf{c}, w, \mathrm{~g})\right)^{\prime}, w^{\prime}\right)=\mathrm{g}^{m}\left(\alpha^{k}(\mathbf{c}, w, \mathrm{~g}), w\right)
$$

where the first and last equalities hold by definition of $g$, and the second equality holds because $f$ satisfies 2-VSP. The third equality follows from Claim A applied to rule $\mathrm{f}$ and markets $\left(\alpha^{k}\left(\mathbf{c}^{\prime}, w^{\prime}, \mathbf{f}\right), w^{\prime}\right)$ and $\left(\left(\alpha^{k}(\mathbf{c}, w, \mathrm{~g})\right)^{\prime}, w^{\prime}\right)$, and is explained as follows: On the one hand, the two markets have the same firms' bargaining power. Indeed,

$$
\begin{aligned}
& \alpha_{2}^{k}\left(\mathbf{c}^{\prime}, w^{\prime}, \mathbf{f}\right)-\alpha_{1}^{k}\left(\mathbf{c}^{\prime}, w^{\prime}, \mathbf{f}\right)=\left(c^{\prime}\right)_{2}^{k}-\left(c^{\prime}\right)_{1}^{k}=c_{2}^{k}-c_{1}^{k}=\alpha_{2}^{k}(\mathbf{c}, w, \mathrm{~g})-\alpha_{1}^{k}(\mathbf{c}, w, \mathrm{~g}) \\
= & \left(\alpha^{k}(\mathbf{c}, w, \mathrm{~g})\right)_{2}^{\prime}-\left(\alpha^{k}(\mathbf{c}, w, \mathrm{~g})\right)_{1}^{\prime}
\end{aligned}
$$

where the first and third equalities are obtained using Eq. (9), and the second and last equalities follow from Eq. (21). On the other hand, using the definition of g, it follows from immediate algebraic manipulations that

$$
\left(w_{1}^{\prime}-\alpha_{1}^{k}\left(\mathbf{c}^{\prime}, w^{\prime}, \mathrm{f}\right)\right)_{+}=\mathrm{f}_{1}^{k}\left(\mathbf{c}^{\prime}, w^{\prime}\right)+\mathrm{f}_{1}^{m}\left(\mathbf{c}^{\prime}, w^{\prime}\right)=\left(w_{1}^{\prime}-\left(\alpha^{k}(\mathbf{c}, w, \mathrm{~g})\right)_{1}^{\prime}\right)_{+} .
$$

Therefore, the surplus of the two markets is the same.

Case B: $(\mathbf{c}, w) \sim^{m}\left(\mathbf{c}^{*}, w^{*}\right)$.

Then,

$$
\mathrm{g}^{m}(\mathbf{c}, w)=\mathrm{f}^{m}\left(\mathbf{c}^{*}, w^{*}\right)=\mathrm{f}^{m}\left(\alpha^{k}\left(\mathbf{c}^{*}, w^{*}, \mathrm{f}\right), w^{*}\right)=\mathrm{f}^{m}\left(\left(\alpha^{k}(\mathbf{c}, w, \mathrm{~g})\right)^{\prime}, w^{\prime}\right)=\mathrm{g}^{m}\left(\alpha^{k}(\mathbf{c}, w, \mathrm{~g}), w\right)
$$

where the first and last equalities hold by definition of $g$, and the second equality holds because $f$ satisfies 2-VSP. The third equality is due to Claim A being applied to rule $\mathbf{f}$ and markets $\left(\alpha^{k}\left(\mathbf{c}^{*}, w^{*}, \mathbf{f}\right), w^{*}\right)$ and $\left(\left(\alpha^{k}(\mathbf{c}, w, \mathrm{~g})\right)^{\prime}, w^{\prime}\right)$, and is explained as follows: On the one hand, both markets have the same firms' bargaining power. Indeed,

$$
\begin{aligned}
\alpha_{2}^{k}\left(\mathbf{c}^{*}, w^{*}, \mathbf{f}\right)-\alpha_{1}^{k}\left(\mathbf{c}^{*}, w^{*}, \mathrm{f}\right) & =\left(c^{*}\right)_{2}^{k}-\left(c^{*}\right)_{1}^{k}=c_{2}^{k}-c_{1}^{k}=\alpha_{2}^{k}(\mathbf{c}, w, \mathrm{~g})-\alpha_{1}^{k}(\mathbf{c}, w, \mathrm{~g}) \\
& =\left(\alpha^{k}(\mathbf{c}, w, \mathrm{~g})\right)_{2}^{\prime}-\left(\alpha^{k}(\mathbf{c}, w, \mathrm{~g})\right)_{1}^{\prime}
\end{aligned}
$$

where the first and third equalities are obtained using Eq. (9), the second equality is due to the fact that $(\mathbf{c}, w) \sim^{m}\left(\mathbf{c}^{*}, w^{*}\right)$, and the fourth equality holds by Eq. (21). Moreover,

$$
w_{1}^{*}-w_{2}^{*}=w_{1}-w_{2}=w_{1}^{\prime}-w_{2}^{\prime},
$$

where the first equality holds because $(\mathbf{c}, w) \sim^{m}\left(\mathbf{c}^{*}, w^{*}\right)$, and the second equality follows from Eq. (21). On the other hand, the surplus of the two markets is the same. Indeed, due to definition of $g$ and Eq. (21), by straightforward algebraic manipulations we obtain

$$
\begin{aligned}
\left(w_{1}^{*}-\alpha_{1}^{k}\left(\mathbf{c}^{*}, w^{*}, \mathbf{f}\right)\right)_{+} & =\mathrm{f}_{1}^{k}\left(\mathbf{c}^{*}, w^{*}\right)+\mathrm{f}_{1}^{m}\left(\mathbf{c}^{*}, w^{*}\right)=\mathrm{g}_{1}^{k}(\mathbf{c}, w)+\mathrm{g}_{1}^{m}(\mathbf{c}, w) \\
& =\left(w_{1}-\alpha_{1}^{k}(\mathbf{c}, w, \mathbf{g})\right)_{+}=\left(w_{1}^{\prime}-\alpha_{1}^{k}(\mathbf{c}, w, \mathbf{g})^{\prime}\right)_{+} .
\end{aligned}
$$


Case C: $(\mathbf{c}, w) \sim^{l}\left(\mathbf{c}^{*}, w^{*}\right)$ for some $l \in M \backslash\{m\}$.

The proof of $\mathrm{g}^{m}(\mathbf{c}, w)=\mathrm{g}^{m}\left(\alpha^{k}(\mathbf{c}, w, \mathrm{~g}), w\right)$ proceeds by showing two chains of equalities, which yield the desired result when appended. The first chain is

$$
\mathrm{g}^{m}(\mathbf{c}, w)=\mathrm{f}^{l}\left(\mathbf{c}^{*}, w^{*}\right)=\mathrm{f}^{l}\left(\alpha^{l}\left(\mathbf{c}^{*}, w^{*}, \mathrm{f}\right), w^{*}\right)=\mathrm{f}^{m}\left(\alpha^{l}(\mathbf{c}, w, \mathfrak{f}), w\right)=\mathrm{f}^{m}(\mathbf{c}, w),
$$

where the first equality holds by definition of $g$, the second equality follows from the fact that $f$ satisfies 2-VSP and CS - and hence Lemma 5.3-, and the last equality holds because $\mathrm{f}$ satisfies 2-VSP. The third equality follows from application of Claim B to rule $f$ and markets $\left(\alpha^{l}\left(\mathbf{c}^{*}, w^{*}, f\right), w^{*}\right)$ and $\left(\alpha^{l}(\mathbf{c}, w, f), w\right)$. Indeed,

$$
w_{1}^{*}-w_{2}^{*}=c_{2}^{l}-c_{1}^{l}=\alpha_{2}^{l}(\mathbf{c}, w, \mathbf{f})-\alpha_{1}^{l}(\mathbf{c}, w, \mathbf{f})
$$

where the first equality holds because $(\mathbf{c}, w) \sim^{l}\left(\mathbf{c}^{*}, w^{*}\right)$ and the second equality follows from Eq. (9). Similarly,

$$
\alpha_{2}^{l}\left(\mathbf{c}^{*}, w^{*}, \mathbf{f}\right)-\alpha_{1}^{l}\left(\mathbf{c}^{*}, w^{*}, \mathbf{f}\right)=\left(c^{*}\right)_{2}^{l}-\left(c^{*}\right)_{1}^{l}=w_{1}-w_{2},
$$

where the first equality follows from Eq. (9) and the second equality holds because (c, $w) \sim^{l}\left(\mathbf{c}^{*}, w^{*}\right)$. That is, both markets have switched bargaining powers. Moreover, the total surplus is equal to $\mathbf{f}_{1}^{m}\left(\mathbf{c}^{*}, w^{*}\right)+\mathbf{f}_{1}^{l}\left(\mathbf{c}^{*}, w^{*}\right)$ and $\mathbf{f}_{1}^{l}(\mathbf{c}, w)+\mathbf{f}_{1}^{m}(\mathbf{c}, w)$, respectively. Since $(\mathbf{c}, w) \sim^{l}\left(\mathbf{c}^{*}, w^{*}\right)$ and $\mathbf{f}$ satisfies 2-ABP, both quantities coincide. On the other hand, the second chain of equalities is

$$
\mathrm{f}^{m}(\mathbf{c}, w)=\mathrm{f}^{m}\left(\alpha^{k}(\mathbf{c}, w, \mathbf{f}), w\right)=\mathrm{f}^{m}\left(\left(\alpha^{k}(\mathbf{c}, w, \mathrm{~g})\right)^{\prime}, w^{\prime}\right)=\mathrm{g}^{m}\left(\alpha^{k}(\mathbf{c}, w, \mathrm{~g}), w\right),
$$

where the first equality holds because $f$ satisfies 2-VSP, the last equality is by definition of $g$, and the second equality is consequence of applying Claim A to rule $\mathbf{f}$ and markets $\left(\alpha^{k}(\mathbf{c}, w, \mathbf{f}), w\right)$ and $\left(\left(\alpha^{k}(\mathbf{c}, w, \mathbf{g})\right)^{\prime}, w^{\prime}\right)$. Indeed, by Eq. (21) it immediately follows that both markets have the same bargaining powers and that their total surplus is equal to $\mathrm{f}_{1}^{k}(\mathbf{c}, w)+\mathrm{f}_{1}^{m}(\mathbf{c}, w)$ and $\mathbf{g}_{1}^{k}(\mathbf{c}, w)+\mathbf{g}_{1}^{m}(\mathbf{c}, w)$, respectively. We next show that the two latter quantities coincide. Together with Eqs. (23) and (24), this will imply that g satisfies 2-VSP. To show the latter claim, we distinguish two cases.

Case C.I: $k=l$. In this case,

$$
\begin{aligned}
\mathrm{g}^{k}(\mathbf{c}, w)+\mathrm{g}^{m}(\mathbf{c}, w) & =\mathrm{g}^{m}\left(\mathbf{c}^{*}, w^{*}\right)+\mathrm{g}^{k}\left(\mathbf{c}^{*}, w^{*}\right)=\mathrm{f}^{m}\left(\mathbf{c}^{*}, w^{*}\right)+\mathrm{f}^{k}\left(\mathbf{c}^{*}, w^{*}\right) \\
& =\mathrm{f}^{k}(\mathbf{c}, w)+\mathrm{f}^{m}(\mathbf{c}, w),
\end{aligned}
$$

where the first two equalities hold by definition of $g$, and the last equality holds since $f$ satisfies $2-\mathrm{ABP}$.

Case C.II: $k \neq l$. In this case,

$$
\begin{aligned}
\mathrm{g}^{k}(\mathbf{c}, w)+\mathrm{g}^{m}(\mathbf{c}, w) & =\mathrm{g}^{k}\left(\mathbf{c}^{*}, w^{*}\right)+\mathrm{g}^{l}\left(\mathbf{c}^{*}, w^{*}\right)=\mathrm{f}^{k}\left(\mathbf{c}^{*}, w^{*}\right)+\mathrm{f}^{l}\left(\mathbf{c}^{*}, w^{*}\right) \\
& =\mathrm{f}^{k}(\mathbf{c}, w)+\mathrm{f}^{m}(\mathbf{c}, w),
\end{aligned}
$$

where the first two equalities hold by definition of $g$, and the last equality holds since $f$ satisfies 2-ABP. 
Finally, since $g$ satisfies the four properties, $f$ and $g$ must coincide in $\mathcal{H}$. In particular,

$$
\mathrm{f}\left(\mathbf{c}^{*}, w^{*}\right)=\mathrm{g}\left(\mathbf{c}^{*}, w^{*}\right)=\mathrm{g}\left(\left(\mathbf{c}^{*}\right)^{\prime},\left(w^{*}\right)^{\prime}\right)=\mathrm{f}\left(\left(\mathbf{c}^{*}\right)^{\prime},\left(w^{*}\right)^{\prime}\right)
$$

where the first equality holds by definition of $\mathrm{g}$, the second is due to the fact that, by Eq. (21), $\left(\left(\mathbf{c}^{*}\right)^{\prime},\left(w^{*}\right)^{\prime}\right) \sim^{m}\left(\mathbf{c}^{*}, w^{*}\right)$, and the third equality holds since $\left(\left(\mathbf{c}^{*}\right)^{\prime},\left(w^{*}\right)^{\prime}\right) \in \mathcal{H}$. As a consequence, Eq. (22) cannot hold, and the proof is complete.

Step 2: Existence

To demonstrate that CS, 2-VSP, 2-ABP, and 2-CBS are compatible properties for rules in $\mathcal{B B} \mathcal{M}$ is straightforward. Indeed, from Theorem 5.1 and Proposition 6.2 it immediately follows that $\mathrm{T}=\Phi(\mathrm{Tb})$ satisfies the four properties.

\section{Step 3: The characterization result}

Given the results in the previous two steps, to prove the theorem it only remains to show that T selects the nucleolus of the associated assignment game. Indeed, by part (ii) of Theorem 2.1 and Theorem I-together with its proof-we know that $\mathrm{T}$ selects for every $(\mathbf{c}, w) \in \mathcal{B B} \mathcal{M}$ the nucleolus of $\left(N, v^{\mathbf{c}, w}\right)$.

Theorem II shows that 2-VSP-together with the other three properties-leads to a solution, T, constructed for every $(\mathbf{c}, w) \in \mathcal{B B} \mathcal{M}$ in two steps. First, the information contained in the rows $r$ and $r+1$ of $(\mathbf{c}, w)$ is used to construct the sectors game, for which the nucleolus is calculated. Second, the information in the remaining rows of $(\mathbf{c}, w)$ is used by the replica operator and the translation vector to obtain the payoffs for all agents of $(\mathbf{c}, w)$.

We make two further remarks. First, as a corollary of Theorem II we obtain that for any rule in $\mathcal{B B} \mathcal{B}, \mathrm{f}$, other than $\mathrm{T}$ there might be incentives for sellers owning different commodities to collude and create a vertical syndicate. Second, no property can be excluded from the characterization of $\mathrm{T}$ in Theorem II-, as shown in Proposition 8.2 in the Appendix.

\section{Other forms of syndication}

In this section, we present one positive result and one impossibility result. First, we show that the Talmud Assignment Rule satisfies a stronger version of 2-VsP. This property applies to general markets and permits vertical syndicates with an arbitrary number of members. Second, we show that among the solution concepts that satisfy 2-CBS, CS, and 2-ABP, none simultaneously satisfies our notions of vertical and horizontal syndication-proofness - see below.

Let us formalize the properties just described. We start with a possible extension of 2-VsP. Given $S \subseteq M \backslash\{m\}$, let $N^{M \backslash(S \cup\{m\})}=\left(N^{l}\right)_{l \in M \backslash(S \cup\{m\})}$ and $\mathbf{c}^{M \backslash(S \cup\{m\})}=\left(c^{l}\right)_{l \in M \backslash(S \cup\{m\})} \cdot{ }^{20}$

SvsP A rule in $\mathcal{B B} \mathcal{M}, \mathrm{f}$, satisfies strong vertical syndication-proofness if for every $(\mathbf{c}, w) \in \mathcal{B B} \mathcal{M}$, every $\emptyset \neq S \subseteq M \backslash\{m\}$, and every $k \in S$, we have that for every $j \in M \backslash S$,

$$
\mathrm{f}^{j}\left(N^{1}, \ldots, N^{m}, \mathbf{c}, w\right)=\mathrm{f}^{j}\left(N^{k}, N^{M \backslash(S \cup\{m\})}, N^{m}, \beta^{k}(\mathbf{c}, w, \mathrm{f}, S), \mathbf{c}^{M \backslash(S \cup\{m\})}, w\right),
$$

\footnotetext{
${ }^{20}$ In line with part $(i)$ of Lemma 5.2, it can be verified that the reduced market in the definition of SVSP is a 2-regular market whenever $\mathrm{f}$ satisfies CS and $(\mathbf{c}, w) \in 2-\mathcal{B B M}$.
} 
where the reduced ( $m-|S|+1)$-sided market in the right-hand side of Eq. (25) is composed of the original sector of buyers, with valuations given by $w$, of the original sectors of sellers $N^{l}$, with $l \in M \backslash(S \cup\{m\})$, with costs given by $\left(c^{l}\right)_{l \in M \backslash(S \cup\{m\})}$, and a new sector of sellers, $N^{k}$, with costs, $\beta^{k}(\mathbf{c}, w, \mathrm{f}, S)$ defined for every $i \in N^{k}$ by

$$
\beta_{i}^{k}(\mathbf{c}, w, \mathrm{f}, S)=c_{i}^{k}+\sum_{l \in S \backslash\{k\}} p^{l}(\mathrm{f}(\mathbf{c}, w)) .
$$

Recall that for the markets under consideration, the interests of all agents in one sector are completely aligned in competitive prices. This implies that there is an incentive to create a vertical syndicate for some sellers if and only if there is an incentive to create a vertical syndicate for all active sellers in the sectors to which the former belong. Accordingly, the restrictions imposed by SVSP are reasonable for our class of markets.

Next, we consider horizontal syndicates.

HSP A rule in $\mathcal{B B M}, \mathrm{f}$, satisfies horizontal syndication-proofness if for every $(\mathbf{c}, w) \in \mathcal{B B M}$, every $k \in M \backslash\{m\}$, and every $\emptyset \neq S \subseteq N^{k}$,

$$
\mathrm{f}^{m}\left(N^{1}, \ldots, N^{m}, \mathbf{c}, w\right)=\mathrm{f}^{m}\left(S,\left(N^{l}\right)_{l \in M \backslash\{k, m\}}, N^{m}, \gamma^{k, S}, \mathbf{c}^{M \backslash\{k, m\}}, w\right),
$$

and

$$
\sum_{i \in N^{k}} \mathrm{f}_{i}^{k}(\mathbf{c}, w) \leq \sum_{i \in S} \mathrm{f}_{i}^{k}\left(\gamma^{k, S}, \mathbf{c}^{M \backslash\{k, m\}}, w\right),
$$

where $\mathbf{c}^{M \backslash\{k, m\}}=\left(c^{l}\right)_{l \in M \backslash\{k, m\}}$ and $\gamma^{k, S}=\left(c_{i}^{k}\right)_{i \in S}{ }^{21}$

The above property, HSP, requires that the benefits assigned by a rule to each of the buyers should not be affected by the horizontal syndicate composed of all sellers in $N^{k}$ when they decide to dump only those goods into the market that belong to the sellers in $S$ - see Eq. (26). We have also assumed that the syndicate behaves in a rational way, i.e., the aggregate payoff to all agents in $N^{k}$ does not decrease - see Eq. (27). Unlike 2-SVP, HSP does not imply that sellers have no incentives to create a (horizontal) syndicate even when prices are competitive. We refer to the property obtained by imposing the requirements in HSP only to 2-regular markets as horizontal syndication-proofness for 2-regular markets and we denote it by 2-HSP.

In the following, we show the results concerning the above two properties.

Proposition 7.1. The Talmud Assignment Rule, T, satisfies SvSP.

Proposition 7.1 reveals that the Talmud Assignment Rule is vertical syndication-proof for all markets, but Theorem II specifies that this stronger property is not necessary to characterize the rule.

Proposition 7.2. Let $\mathrm{f}$ be a rule in $\mathcal{B B M}$ that satisfies $\mathrm{CS}, 2-\mathrm{CBS}$, and 2-ABP. Then $\mathrm{f}$ does not satisfy simultaneously 2-VSP and 2-HSP.

Proposition 7.2 identifies certain conditions under which, for the market games considered in this paper and also for any superclass containing them, a rule does not exist that specifies prices so that buyers are immune to any arbitrary syndication of the sellers.

\footnotetext{
${ }^{21}$ To be consistent with the assumptions made throughout this paper, dummy sellers might be added to sector $k$ so that there is the same number of agents in all sectors.
} 


\section{Conclusion}

This paper has considered syndication in markets. In the literature, various attempts at studying this phenomenon have been framed within cooperative game theory. Here, we have opted for one particular cooperative-game solution concept, viz. the nucleolus (see Legros, 1987; Einy et al., 1999). We believe that it holds out particular promise for an improved understanding of the formation and behavior of syndicates.

The market games considered herein belong to the class of multi-sided assignment games. The literature on the latter is relatively scarce, and mainly focuses on finding sufficient conditions to guarantee the non-emptiness of the core (see Quint, 1991; Stuart Jr, 1997; Sherstyuk, 1999). Our findings look beyond this concern and yield insights into the functioning of these games.

Multi-sided assignment games with more than two types of agents enable the insightful investigation of vertical syndicates. We have proposed a definition of vertical syndication-proofness for these market games. Such a property may be relevant to a regulator who has to decide whether to allow this form of integration in particular branches of the economy.

Actual markets exist such that (i) their economic structure resembles that of a (Böhm-Bawerk) multi-sided assignment market and (ii) their vertical production chain has caused some concern. In this regard, we have discussed the IT industry in the Introduction. Vertical integration in the steel industry, whose products are largely homogeneous, is also both practiced and the object of regulators' attention. The European Union, for example, imposes restrictions on the conduct of the various parties by means of antitrust laws. ${ }^{22}$

Lastly, this paper has also established a connection between multi-sided assignment games and bankruptcy games. Exploiting this connection appears to be a promising direction for future research.

\section{Acknowledgments}

This research received financial support from the Ministerio de Economía y Competitividad through Projects ECO2014-52340-P and MTM2014-53395-C3-2-P as well as from Generalitat de Catalunya through project 2014-SGR-40. Discussions with Julio González-Díaz, Hans Gersbach, Marie Riekhof, and Clive Bell improved the paper and are gratefully acknowledged. We also acknowledge the comments received at the seminars and conferences in which this work was presented: Seminars at the Hebrew University of Jerusalem, Universitat de Barcelona, and University of Southern Denmark as well as at the SING9 and IWGTS2014 conferences in Vigo and São Paulo, respectively. Last but not least, we would like to thank the referees for their comments and suggestions which substantially helped improve a previous version of the paper. Finally, the usual disclaimer applies.

\section{References}

Asch, P. and Seneca, J. (1976). Is collusion profitable? Review of Economics and Statistics, 58:1-12.

\footnotetext{
${ }^{22}$ See http://ec.europa.eu/competition/antitrust/legislation/legislation.html, retrieved on 10-September2016.
} 
Aumann, R. (1973). Disadvantageous monopolies. Journal of Economic Theory, 6:1-11.

Aumann, R. and Maschler, M. (1985). Game theoretic analysis of a bankruptcy problem from the Talmud. Journal of Economic Theory, 20:455-481.

Bloch, F. and Ghosal, S. (2000). Buyers' and sellers' cartels on markets with indivisible goods. Review of Economic Design, 5:129-147.

Brandenburger, A. and Stuart, H. (2007). Biform games. Management science, 53:537-549.

Dagan, N. (1996). New characterizations of old bankruptcy rules. Social Choice and Welfare, 13:5159.

Dubey, P., Mas-Colell, A., and Shubik, M. (1980). Efficiency properties of strategies market games: An axiomatic approach. Journal of Economic Theory, 22:339-362.

Economides, N. (2001). The Microsoft antitrust case. Journal of Industry, Competition and Trade, $1: 7-39$.

Einy, E., Moreno, D., and Shitovitz, B. (1999). The asymptotic nucleolus of large monopolistic market games. Journal of Economic Theory, 89:186-206.

Fershtman, C. and Gandal, N. (1994). Disadvantageous semicollusion. International Journal of Industrial Organization, 12:141-154.

Gale, D. (1960). The theory of linear economic models. University of Chicago Press.

Gardner, R. (1977). Shapley value and disadvantageous monopolies. Journal of Economic Theory, $16: 513-517$.

González-Díaz, J. and Sánchez-Rodríguez, E. (2007). A natural selection from the core of a TU game: the core-center. International Journal of Game Theory, 36:27-46.

Granot, D. and Granot, F. (1992). On some network flow games. Mathematics of Operations Research, $17: 792-841$.

Guesnerie, R. (1977). Monopoly, syndicate, and shapley value: About some conjectures. Journal of Economic Theory, 15:235-251.

Herrero, C. and Villar, A. (2001). The three musketeers: Four classical solutions to bankruptcy problems. Mathematical Social Sciences, 42:307-328.

Inderst, R. and Wey, C. (2007). Buyer power and supplier incentives. European Economic Review, 51:647-667.

Kaneko, M. and Wooders, M. H. (1982). Cores of partitioning games. Mathematical Social Sciences, $3: 313-327$.

Knudsen, P. H. and Østerdal, L. P. (2012). Merging and splitting in cooperative games: some (im)possibility results. International Journal of Game Theory, 41:763-774. 
Legros, P. (1987). Disadvantageous syndicates and stable cartels: The case of the nucleolus. Journal of Economic Theory, 42:30-49.

Mendelson, H. (1982). Market behavior in a clearing house. Econometrica, 50:1505-1524.

Milliou, C. and Petrakis, E. (2007). Upstream horizontal mergers, vertical contracts, and bargaining. International Journal of Industrial Organization, 25:963-987.

Moreno-Ternero, J. D. and Villar, A. (2006). New characterizations of a classical bankruptcy rule. Review of Economic Design, 10:73-84.

Nash Jr, J. F. (1950). The bargaining problem. Econometrica, 18:155-162.

Núñez, M. and Rafels, C. (2005). The Böhm-Bawerk horse market: A cooperative analysis. International Journal of Game Theory, 33:421-430.

Okuno, M., Postlewaite, A., and Roberts, J. (1980). Oligopoly and competition in large markets. American Economic Review, 70:22-31.

O'Neill, B. (1982). A problem of rights arbitration from the Talmud. Mathematical Social Sciences, 2:345-371.

Postlewaite, A. and Rosenthal, R. (1974). Disadvantageous syndicates. Journal of Economic Theory, 9:324-326.

Quint, T. (1991). The core of an m-sided assignment game. Games and Economic Behavior, 3:487503.

Salant, S., Switzer, S., and Reynolds, R. (1983). Losses from horizontal merger: the effects of an exogenous change in industry structure on Cournot-Nash equilibrium. The Quarterly Journal of Economics, 98:185-199.

Schmalensee, R. (2007). Handbook of industrial organization, volume 3. Elsevier.

Schmeidler, D. (1969). The nucleolus of a characteristic function game. SIAM Journal on Applied Mathematics, 17:1163-1170.

Segal, I. (2003). Collusion, exclusion, and inclusion in random-order bargaining. The Review of Economic Studies, 70:439-460.

Shapley, L. and Shubik, M. (1972). The assignment game I: the core. International Journal of Game Theory, 1:11-26.

Sherstyuk, K. (1999). Multisided matching games with complementarities. International Journal of Game Theory, 28:489-509.

Sproul, M. F. (1993). Antitrust and prices. Journal of Political Economy, 101:741-754.

Stuart Jr, H. W. (1997). The supplier-firm-buyer game and its m-sided generalization. Mathematical Social Sciences, 34:21-27. 
Tejada, O. (2010). A note on competitive prices in multilateral assignment markets. Economics Bulletin, 30:658-662.

Tejada, O. (2013). Analysis of the core of multisided Böhm-Bawerk assignment markets. TOP, 21:1-17.

Tejada, O. and Núñez, M. (2012). The nucleolus and the core-center of multi-sided Böhm-Bawerk assignment markets. Mathematical Methods of Operations Research, 75:199-220.

Thomson, W. (2003). Axiomatic and game theoretic analysis of bankruptcy and taxation problems: A survey. Mathematical Social Sciences, 45:249-297.

Thomson, W. (2015). Axiomatic and game-theoretic analysis of bankruptcy and taxation problems: An update. Mathematical Social Sciences, 74:41-59.

von Böhm-Bawerk, E. (1930). Positive theory of capital (translated by W. Smart). G.E. Stechert \& co., New York, NY, (original publication 1891). 


\section{Appendix}

Proposition 8.1. The set of assignment games associated with 2-regular markets, 2-BBG , and the set of sectors games, $\mathcal{S G}$, are isomorphic, i.e.,

$$
2-\mathcal{B B G} \cong \mathcal{S G} .
$$

Proof of Proposition 8.1. Let $N^{1}, \ldots, N^{m}$ be fixed throughout the proof, and recall that $M=\{1, \ldots, m\}$ and $N=N^{1} \cup \cdots \cup N^{m}$. First, note that due to Remark 2.2,

$$
\mathcal{S G}=\left\{\left(M, v^{\mathbf{c}, w}\right):(\mathbf{c}, w) \in 2-\mathcal{B B} \mathcal{M}\right\}
$$

and, by Definition 2.3,

$$
{ }_{2}-\mathcal{B B G}=\left\{\left(N, \omega^{\mathbf{c}, w}\right):(\mathbf{c}, w) \in 2-\mathcal{B B M}\right\} .
$$

Second, consider the following mapping:

$$
\begin{aligned}
\Gamma: \quad 2-\mathcal{B B G} & \rightarrow \mathcal{S G} \\
\left(N, \omega^{\mathbf{c}, w}\right) & \rightarrow\left(M, v^{\mathbf{c}, w}\right)
\end{aligned}
$$

Note that if $\left(N, \omega^{\mathbf{c}, w}\right)=\left(N, \omega^{\mathbf{c}^{\prime}, w^{\prime}}\right)$, then $\left(M, v^{\mathbf{c}, w}\right)=\left(M, v^{\mathbf{c}^{\prime}, w^{\prime}}\right)$, so $\Gamma$ is well defined. Moreover, it is straightforward to check that $\Psi$ is surjective. To check that it is also injective, let $\left(N, \omega^{\mathbf{c}, w}\right),\left(N, \omega^{\mathbf{c}^{\prime}, w^{\prime}}\right) \in 2-\mathcal{B B G}$ be such that

$$
\omega^{\mathbf{c}, w} \neq \omega^{\mathbf{c}^{\prime}, w^{\prime}}
$$

On the one hand, suppose that $r(\mathbf{c}, w)=0$. Then, by Eq. (28) it must be that $r\left(\mathbf{c}^{\prime}, w^{\prime}\right)=1$. Thus, $v^{\mathbf{c}, w}=v_{0}$ and $v^{\mathbf{c}^{\prime}, w^{\prime}} \neq v_{0}$, which implies $\Gamma\left(N, \omega^{\mathbf{c}, w}\right) \neq \Gamma\left(N, \omega^{\mathbf{c}^{\prime}, w^{\prime}}\right)$.

On the other hand, assume that $r(\mathbf{c}, w)=r\left(\mathbf{c}^{\prime}, w^{\prime}\right)=1$. From Eq. (28) it follows the existence of $S \subseteq N$ minimal w.r.t. to inclusion such that $\omega^{\mathbf{c}, w}(S) \neq \omega^{\mathbf{c}^{\prime}, w^{\prime}}(S)$. Furthermore, from the definition of the characteristic function of an assignment game, it must be that $S=Z^{R}$ for some $R \subseteq M$. Hence,

$$
v^{\mathbf{c}, w}(R)=\omega^{\mathbf{c}, w}(S) \neq \omega^{\mathbf{c}^{\prime}, w^{\prime}}(S)=v^{\mathbf{c}^{\prime}, w^{\prime}}(R) .
$$

Thus, $\Gamma\left(N, \omega^{\mathbf{c}, w}\right) \neq \Gamma\left(N, \omega^{\mathbf{c}^{\prime}, w^{\prime}}\right)$, so $\Gamma$ is injective.

Proof of Lemma 5.1. Let $\mathrm{f}$ be a rule in $\mathcal{B B M}$ satisfying CS, then it trivially satisfies part (ii). Part (i) then follows from Theorem 2.1. The reverse implication follows from Theorem 2.1 and the properties of the market $(\tilde{\mathbf{c}}, \tilde{w})$ defined in Eq. (2).

Proof of Lemma 5.2. We only prove the first part, as the second part is trivial. Indeed, $\left|N^{k}\right|=\left|N^{m}\right|=2$ since $(\mathbf{c}, w) \in 2-\mathcal{B B M}$, and

$$
\begin{aligned}
& w_{2}-\alpha_{2}^{k}(\mathbf{c}, w, f) \\
= & w_{2}-c_{2}^{k}-\sum_{l \in M \backslash\{k, m\}}\left(f_{1}^{l}(\mathbf{c}, w)+c_{1}^{l}\right)=w_{2}-c_{2}^{k}-\sum_{l \in M \backslash\{k, m\}} c_{1}^{l}-\sum_{l \in M \backslash\{k, m\}} f_{1}^{l}(\mathbf{c}, w) \\
\leq & \left(w_{2}-c_{2}^{k}-\sum_{l \in M \backslash\{k, m\}} c_{1}^{l}\right)_{+}-\left[\mathrm{f}_{2}^{k}(\mathbf{c}, w)+\mathrm{f}_{2}^{m}(\mathbf{c}, w)+\sum_{l \in M \backslash\{k, m\}} f_{1}^{l}(\mathbf{c}, w)\right] \leq 0,
\end{aligned}
$$


where the penultimate inequality holds because $\mathrm{f}_{2}^{k}(\mathbf{c}, w)=\mathrm{f}_{2}^{m}(\mathbf{c}, w)=0$ - due to Theorem 2.1-, the fact that $(\mathbf{c}, w)$ is a 2-regular market, and because $\mathbf{f}$ satisfies $\mathrm{CS}$, while the last inequality holds since $\left(\mathrm{f}_{k}(\mathbf{c}, w)\right)_{k \in M}$ is a core allocation of $\left(N, v^{\mathbf{c}, w}\right)$.

Proof of Lemma 5.3. By Lemma 5.2, the reduced market is a 2-regular market, so $r\left(\alpha^{k}(\mathbf{c}, w, \mathbf{f}), w\right) \leq 1$. Then it suffices to realize that CS implies $\mathbf{f}_{2}^{k}(\mathbf{c}, w)=\mathbf{f}_{2}^{k}\left(\alpha^{k}(\mathbf{c}, w, \mathbf{f}), w\right)=0$ and

$$
\begin{aligned}
\mathrm{f}_{1}^{k}(\mathbf{c}, w) & =\omega^{\mathbf{c}, w}(N)-\sum_{l \in M \backslash\{k, m\}} \mathrm{f}_{1}^{l}(\mathbf{c}, w)-\mathrm{f}_{1}^{m}(\mathbf{c}, w) \\
& =\omega^{\mathbf{c}, w}(N)-\sum_{l \in M \backslash\{k, m\}} \mathrm{f}_{1}^{l}(\mathbf{c}, w)-\mathrm{f}_{1}^{m}\left(\alpha^{k}(\mathbf{c}, w, \mathbf{f}), w\right) \\
& =\omega^{\mathbf{c}, w}(N)-\sum_{l \in M \backslash\{k, m\}} \mathrm{f}_{1}^{l}(\mathbf{c}, w)-\omega^{\alpha^{k}(\mathbf{c}, w, \mathbf{f}), w}\left(N^{k} \cup N^{m}\right)+\mathrm{f}_{1}^{k}\left(\alpha^{k}(\mathbf{c}, w, \mathbf{f}), w\right) \\
& =\mathrm{f}_{1}^{k}\left(\alpha^{k}(\mathbf{c}, w, \mathrm{f}), w\right),
\end{aligned}
$$

where the first and the third equalities hold since $\mathrm{f}$ yields efficient allocations for $(\mathbf{c}, w)$ and $\left(\alpha^{k}(\mathbf{c}, w, f), w\right)$ respectively, the second equality holds since $\mathbf{f}$ satisfies 2-vsP, and the last equality holds since by definition of the reduced market,

$$
\begin{aligned}
\omega^{\alpha^{k}(\mathbf{c}, w, \mathbf{f}), w}\left(N^{k} \cup N^{m}\right) & =\left(w_{1}-\alpha_{1}^{k}(\mathbf{c}, w, \mathbf{f})\right)_{+}=\left(w_{1}-\sum_{l \in M \backslash\{m\}} c_{1}^{l}-\sum_{l \in M \backslash\{k, m\}} f_{1}^{l}(\mathbf{c}, w)\right) \\
& =\omega^{\mathbf{c}, w}(N)-\sum_{l \in M \backslash\{k, m\}} f_{1}^{l}(\mathbf{c}, w) .
\end{aligned}
$$

Proof of Claim A. Let $\mathrm{f}$ be a rule in $2-\mathcal{B B M}$ satisfying 2 -CBS and let $(\mathbf{c}, w),(\overline{\mathbf{c}}, \bar{w}) \in 2-\mathcal{B B M}$ be two 2 -sided markets satisfying $w_{1}-c_{1}^{1}=\bar{w}_{1}-\bar{c}_{1}^{1}$ (same total surplus), $c_{2}^{1}-c_{1}^{1}=\bar{c}_{2}^{1}-\bar{c}_{1}^{1}$, and $w_{1}-w_{2}=\bar{w}_{1}-\bar{w}_{2}$ (same bargaining power). Then,

$$
\begin{aligned}
& \bar{w}_{2}-\bar{c}_{1}^{1}=\left(\bar{w}_{1}-w_{1}+w_{2}\right)-\bar{c}_{1}^{1}=\left(\bar{w}_{1}-\bar{c}_{1}^{1}\right)-\left(w_{1}-c_{1}^{1}\right)+\left(w_{2}-c_{1}^{1}\right)=w_{2}-c_{1}^{1}, \\
& \bar{w}_{1}-\bar{c}_{2}^{1}=\bar{w}_{1}-\left(c_{2}^{1}-c_{1}^{1}+\bar{c}_{1}^{1}\right)=\left(\bar{w}_{1}-\bar{c}_{1}^{1}\right)-\left(w_{1}-c_{1}^{1}\right)+\left(w_{1}-c_{2}^{1}\right)=w_{1}-c_{2}^{1} .
\end{aligned}
$$

Since $\mathrm{f}$ satisfies 2-CBS, we obtain $\mathrm{f}(\mathbf{c}, w)=\mathrm{f}(\overline{\mathbf{c}}, \bar{w})$ by inserting the above equations into the two expressions of Eq. (11).

Proof of Claim B. Let $\mathrm{f}$ be a rule in $2-\mathcal{B B M}$ satisfying 2 -CBS and let $(\mathbf{c}, w),(\overline{\mathbf{c}}, \bar{w}) \in 2-\mathcal{B B M}$ be two 2 -sided markets satisfying $w_{1}-c_{1}^{1}=\bar{w}_{1}-\bar{c}_{1}^{1}$ (same total surplus), $c_{2}^{1}-c_{1}^{1}=\bar{w}_{1}-\bar{w}_{2}$, and $w_{1}-w_{2}=\bar{c}_{2}^{1}-\bar{c}_{1}^{1}$ (switched bargaining power). Then,

$$
\begin{aligned}
& \bar{w}_{2}-\bar{c}_{1}^{1}=\left(\bar{w}_{1}-c_{2}^{1}+c_{1}^{1}\right)-\left(\bar{w}_{1}-w_{1}+c_{1}^{1}\right)=w_{1}-c_{2}^{1}, \\
& \bar{w}_{1}-\bar{c}_{2}^{1}=\left(w_{1}-c_{1}^{1}+\bar{c}_{1}^{1}\right)-\left(w_{1}-w_{2}+\bar{c}_{1}^{1}\right)=w_{2}-c_{1}^{1} .
\end{aligned}
$$

Since $\mathrm{f}$ satisfies 2-CBS, we obtain $\mathrm{f}^{1}(\overline{\mathbf{c}}, \bar{w})=\mathrm{f}^{2}(\mathbf{c}, w)$ and $\mathrm{f}^{2}(\overline{\mathbf{c}}, \bar{w})=\mathrm{f}^{1}(\mathbf{c}, w)$ by inserting the above equations into the two expressions of Eq. (11).

Proposition 8.2. The properties that characterize the Talmud Assignment Rule, T, are logically independent. 
Proof. We consider the following four rules in $\mathcal{B B M}$ :

(i) Let the rule in $\mathcal{B B M}, \hat{\mathrm{T}}$, be defined for every $(\mathbf{c}, w) \in \mathcal{B B M}$ as

$$
\hat{\mathbf{T}}(\mathbf{c}, w)= \begin{cases}\mathrm{T}(\mathbf{c}, w) & \text { if }(\mathbf{c}, w) \in 2-\mathcal{B B M}, \\ \mathbf{0} & \text { otherwise. }\end{cases}
$$

Then, $\hat{\mathrm{T}}$ satisfies 2-VSP, 2-ABP, and 2-CBS but not CS.

(ii) Let the rule in $\mathcal{B B M}, \hat{\mathrm{T}}$, assign for every $(\mathbf{c}, w) \in \mathcal{B B M}$ the core-center (see González-Díaz and Sánchez-Rodríguez (2007)) of $\left(N, \omega^{\mathbf{c}, w}\right)$. From Núñez and Rafels (2005) and Tejada and Núñez (2012), we know that this allocation differs from the nucleolus (of the assignment game) for the whole class of market games in $\mathcal{B B G}$, but that they coincide for bilateral markets. Lastly, the core-center is an anonymous solution concept. Then, $\hat{\mathrm{T}}$ satisfies CS, 2-ABP, and 2-CBS, but not 2-VSP.

(iii) Let $C E A$ be the constrained equal awards bankruptcy rule. This rule is defined for every $(M, E, d) \in \mathcal{B P}$ and $i \in M$ by $C E A_{i}(M, E, d)=\min \left\{d_{i}, \lambda\right\}$, where $\lambda$ is chosen so that $\sum_{i \in M} C E A_{i}(E, d)=E$. The following properties are known:

(a) $C E A$ chooses a core allocation of the bankruptcy game $\left(M, v^{E, d}\right)$ - see Theorem 2 in Thomson (2003).

(b) CEA satisfies BC - see Theorem 1 in Herrero and Villar (2001).

(c) $C E A$ does not satisfy CG-by Theorem 5.1 and because $C E A$ satisfies wD by construction.

First, let $\hat{\mathrm{T}}=\Phi(C E A)$. From Theorems 2.1 and I and statement (a), it holds that $\hat{\mathrm{T}}$ satisfies CS. Second, following the same lines as in the proof of statement (ii) in Proposition 6.2, we can show that due to statements (a) and (b), $\hat{\mathrm{T}}$ also satisfies 2-VSP. Third, the definition of $\hat{\mathrm{T}}$ directly implies that it satisfies 2-ABP. Fourth and last, statement (c), statement (ii) in Proposition 6.1, statement (i) in Proposition 6.2, and the fact that $C E A=\Psi(\Phi(C E A))$ imply that $\hat{\mathrm{T}}$ does not satisfy 2-CBS.

(iv) Consider the following subset of $2-\mathcal{B B M}$ :

$$
\mathcal{V}=\left\{\left(\left(c^{1}, c^{2}\right), w\right) \in \mathcal{B B} \mathcal{M}: c_{1}^{1}=c_{1}^{2}=0, c_{2}^{1}=c_{2}^{2}=c, w_{1}=w_{2}<c\right\}
$$

Since $w_{2}-2 c<0$ for all $(\mathbf{c}, w) \in \mathcal{V}$, we have $\mathcal{V} \subseteq 2-\mathcal{B B M}$. Let the rule in $\mathcal{B B M}$, $\hat{\mathrm{T}}$, be such that for every $(\mathbf{c}, w) \in \mathcal{V}$,

$$
x_{k}=\hat{\mathrm{T}}_{1}^{k}\left(N^{1}, N^{2}, N^{3}, \mathbf{c}, w\right)= \begin{cases}w_{1} & \text { if } k=1, \\ 0 & \text { if } k=2, \\ 0 & \text { if } k=3,\end{cases}
$$

and $\hat{\mathbf{T}}_{2}^{k}(\mathbf{c}, w)=0$ for all $k \in M=\{1,2,3\}$. Note that for every $(\mathbf{c}, w) \in \mathcal{V}$

$$
\begin{array}{lll}
v^{\mathbf{c}, w}(\{1\})=\left(w_{2}-c\right)_{+}=0, & v^{\mathbf{c}, w}(\{1,2\})=\left(w_{2}\right)_{+}=w_{2}, \\
v^{\mathbf{c}, w}(\{2\})=\left(w_{2}-c\right)_{+}=0, & v^{\mathbf{c}, w}(\{1,3\})=\left(w_{1}-c\right)_{+}=0, & v^{\mathbf{c}, w}(\{1,2,3\})=w_{1} . \\
v^{\mathbf{c}, w}(\{3\})=\left(w_{1}-2 c\right)_{+}=0, & v^{\mathbf{c}, w}(\{2,3\})=\left(w_{1}-c\right)_{+}=0,
\end{array}
$$

Hence, sectors 1 and 2 are symmetric players in the sectors game. Since the nucleolus is an anonymous solution, $\mathbf{T}_{1}^{1}(\mathbf{c}, w)=\mathbf{T}_{1}^{2}(\mathbf{c}, w)$. Also, note that $x_{1}+x_{2}=w_{1} \geq w_{1}$, so $x \in C\left(M, v^{\mathbf{c}, w}\right)$, with 
$M=\{1,2,3\}$. It then follows from part (i) of Theorem 2.1 that $\hat{\mathbf{T}}(\mathbf{c}, w) \in C\left(N, \omega^{\mathbf{c}, w}\right)$ for all $(\mathbf{c}, w) \in \mathcal{V}$. Next, let the rule in $\mathcal{B B M}, \tilde{\mathrm{T}}$, be defined for every $(\mathbf{c}, w) \in \mathcal{B B M}$ by

$$
\tilde{\mathbf{T}}(\mathbf{c}, w)= \begin{cases}\hat{\mathbf{T}}(\mathbf{c}, w) & \text { if }(\mathbf{c}, w) \in \mathcal{V}, \\ \mathbf{T}(\mathbf{c}, w) & \text { otherwise }\end{cases}
$$

By construction, $\tilde{T}$ satisfies 2-CBS and CS. Next we focus on 2-VSP. On the one hand, consider the reduced market $\left(N^{1}, N^{3}, \alpha^{1}(\mathbf{c}, w, \tilde{\mathbf{T}}), w\right)$, where $\alpha_{1}^{1}(\mathbf{c}, w, \tilde{\mathbf{T}})=0$ and $\alpha_{2}^{1}(\mathbf{c}, w, \tilde{\mathbf{T}})=c$. Then,

$$
\tilde{\mathbf{T}}_{1}^{3}\left(N^{1}, N^{3}, \alpha^{1}(\mathbf{c}, w, \tilde{\mathbf{T}}), w\right)=\frac{w_{1}-0+\left(w_{1}-c\right)_{+}-\left(w_{2}-0\right)_{+}}{2}=0=\tilde{\mathbf{T}}_{1}^{3}\left(N^{1}, N^{2}, N^{3}, \mathbf{c}, w\right) .
$$

On the other hand, consider the reduced game $\left(N^{2}, N^{3}, \alpha^{2}(\mathbf{c}, w, \tilde{\mathrm{T}}), w\right)$, where $\alpha_{1}^{2}(\mathbf{c}, w, \tilde{\mathrm{T}})=w_{1}$ and $\alpha_{2}^{2}(\mathbf{c}, w, \tilde{\mathbf{T}})=w_{1}+c$. Then,

$$
\begin{aligned}
\tilde{\mathbf{T}}_{1}^{3}\left(N^{2}, N^{3}, \alpha^{1}(\mathbf{c}, w, \tilde{\mathbf{T}}), w\right) & =\frac{w_{1}-w_{1}+\left(w_{1}-w_{1}-c\right)_{+}-\left(w_{2}-w_{1}\right)_{+}}{2}=0 \\
& =\tilde{\mathbf{T}}_{1}^{3}\left(N^{1}, N^{2}, N^{3}, \mathbf{c}, w\right) .
\end{aligned}
$$

That is, we have proved that $\tilde{\mathrm{T}}$ satisfies 2-VsP. Lastly, since $\tilde{\mathrm{T}}$ differs from $\mathrm{T}$, it follows from Theorem II that $\tilde{T}$ cannot satisfy 2 -ABP.

Proof of Proposition 7.1. First, we show that T satisfies SVSP for 2-regular markets. Indeed, for such a market, the conditions in the definition of SVSP easily follow by repeating the lines in the proof of Proposition 6.2 and the existence part of Theorem II using consistency instead of bilateral consistency. Finally, from Lemma 5.1 it follows that T satisfies SvsP for arbitrary markets.

Proof of Proposition 7.2. First, note that if a rule in $\mathcal{B B M}$ satisfies 2-CBS, CS, 2-ABP, and 2-VSP, by Theorem II it has to coincide with the Talmud Assignment Rule. Second, consider the bilateral 2-regular market $(\mathbf{c}, w)$ defined by $\mathbf{c}=c^{1}=(1,2)$ and $w=(3,0)$, and let $k=1$ and $S=\{1\} \subseteq N^{1}$. Then, by Definition 6.1,

$$
\mathrm{T}_{1}^{1}\left(c^{1}, w\right)+\mathrm{T}_{2}^{1}\left(c^{1}, w\right)=\frac{1}{2} \leq 1=\mathrm{T}_{1}^{1}\left(\gamma^{1, S}, w\right)+\mathrm{T}_{2}^{1}\left(\gamma^{1, S}, w\right),
$$

and

$$
\mathrm{T}^{2}\left(c^{1}, w\right)=\left(\frac{3}{2}, 0\right) \neq(1,0)=\mathrm{T}^{2}\left(\gamma^{1, S}, w\right),
$$

which concludes the proof. 